Research Article

\title{
Beneficial Effects of Qingzixiaoban Granule on Henoch-Schönlein Purpura Nephritis Mice through Inhibiting Immune Complex Deposition and Th2 Immunodeviation
}

\author{
Hui Yang $\mathbb{D}^{1},{ }^{1}$ Jing Guan, ${ }^{2}$ Pei Ma, ${ }^{1}$ Yannan Fan, ${ }^{1}$ Jinye Bai, ${ }^{1}$ Shuyi Li, ${ }^{1}$ Jiqiao Yuan, \\ Yecheng Jin, ${ }^{3}$ Mingbao Lin $\left(\mathbb{B},{ }^{1}\right.$ Jianmin Zhang $\mathbb{1}^{2},{ }^{2}$ and Qi Hou $\mathbb{1}^{1}$ \\ ${ }^{1}$ State Key Laboratory of Bioactive Substances and Functions of Natural Medicines, Institute of Materia Medica, \\ Chinese Academy of Medical Sciences \& Peking Union Medical College, Beijing, China \\ ${ }^{2}$ Capital Institute of Pediatrics, Beijing, China \\ ${ }^{3}$ Affiliated Sir Run Run Shaw Hospital, Zhejiang University School of Medicine, Hangzhou, China
}

Correspondence should be addressed to Mingbao Lin; mingbaolin@imm.cams.cn, Jianmin Zhang; zjm051525@126.com, and Qi Hou; houq@imm.ac.cn

Received 14 May 2019; Revised 30 August 2019; Accepted 6 September 2019; Published 16 October 2019

Academic Editor: Silvia Wein

Copyright $\odot 2019$ Hui Yang et al. This is an open access article distributed under the Creative Commons Attribution License, which permits unrestricted use, distribution, and reproduction in any medium, provided the original work is properly cited.

\begin{abstract}
Background. Henoch-Schönlein purpura nephritis (HSPN) is the principal cause of morbidity and mortality in HenochSchönlein purpura (HSP). However, there is no absolute consensus for the best management of severe HSPN till now. Qingzixiaoban Granule (QZXB GR), a traditional Chinese medicine formula, has been applied to treat HSP in clinical in China. However, the therapeutic effects and potential mechanism of QZXB GR on HSPN is still unknown. Methods. A Gliadin plus Indian Ink-induced HSPN mice model was established. Renal histopathologic changes and the subcutaneous hemorrhage on left legs were assessed. Hematuria and proteinuria were determined using hemocytometer and bicinchoninic acid assay, respectively. The serum circular immune complex and interleukin- 6 were quantified by ELISA. Using blood biochemical analyzer, the renal biochemical parameters, including serum total protein, albumin, creatinine, and blood urea nitrogen, were measured. The deposition of immune complex in renal tissues and the lymphocyte subsets in peripheral blood and spleen was investigated by immunohistochemistry and flow cytometry. Results. QZXB GR treatment significantly ameliorated renal injury in HSPN mice, by attenuating renal histopathological changes, reducing subcutaneous hemorrhage, decreasing proteinuria/hematuria, regulating renal biochemical parameters, and inhibiting the release of serum interleukin-6. Furthermore, QZXB GR treatment significantly decreased the level of serum circular immune complex, decreased immune complex IgA and IgG deposition in renal tissue, and suppressed Th2 immunodeviation. Conclusion. QZXB GR could prevent renal injury in HSPN mice, and its renoprotective mechanism might be exerted partly through suppressing immune complexes deposition and Th2 immune deviation.
\end{abstract}

\section{Introduction}

Henoch-Schönlein purpura (HSP) nephritis (HSPN) is one of the major clinical manifestations (renal injury) and primary cause of mortality and morbidity in HSP [1]. Within 4-6 weeks after initial disease onset, approximately $30-50 \%$ of children with HSP progress to HSPN [2], which accounts for $1.8-3 \%$ of children with chronic kidney disease and may result in chronic renal failure in $11-38 \%$ of patients with severe manifestations and pathologic changes in long-term follow-up [3]. The severity of renal injury is the key factor determining the prognosis of HSPN [1]. Therefore, great efforts are in urgent need for renal injury controlling in children with HSPN. However, till now, there is no absolute consensus for the best management of severe HSPN, and the most effective treatment remains controversial [3]. Furthermore, corticosteroids, immunosuppressants, and anticoagulants have potential side effects, such as oncogenesis, myelosuppression, hemorrhagic cystitis, and interstitial pneumonia [4]. 
As to this, traditional Chinese medicine (TCM) has shown significant efficacy and advantage in clinical [4] and seems to be an important and novel therapeutic candidate for the treatment of HSPN. In recent years, it has been reported that there were additional positive effects in quite a few trials conducted in China by the use of TCM in conjunction with corticosteroids or immunosuppressive drugs $[5,6]$. Many TCM can improve immune function and reduce the associated renal damage through regulating immune balance and remitting hypercoagulability of blood [7].

Qingzixiaoban Granule (QZXB GR), a formula comes from clinical experience for treating HSP in children and adolescents in China, consists of Indigofera tinctoria L., Lithospermum erythrorhizon Siebold \& Zucc, Salvia miltiorrhiza Bunge, Moutan officinalis (L.) Lindl. \& Paxton, Clematis chinensis Osbeck, Agrimonia pilosa Ledeb., Kochia scoparia (L.) Schrad., and Dictamnus dasycarpus Turcz. Based on the traditional Chinese medicine theory, the beneficial effects of QZXB GR are related to promote blood circulation and remove blood stasis [8]. However, there are limited data regarding therapeutic effects of QZXB GR on HSPN, even lack of potential mechanism data.

Sometimes, HSPN is referred as immunoglobulin (Ig)A vasculitis or anaphylactoid purpura nephropathy, which tends to present as acute glomerular inflammatory lesions resulted from the glomerular deposition of an abnormally glycosylated IgA1, leading to mesangial proliferative changes [9]. Polyclonal B cells are activated with an increase in IgAcontaining complexes that deposit in glomerular mesentery, resulting in mesangial hypercellularity inflammatory cytokine release and extracellular matrix expansion [10] and/or deposit in the small vessels to affect complement activation, increase permeability of vessel wall, and aggravate vascular inflammation [11]; these deposits finally lead to glomeruli and tubules damage [12]. Additionally, the deposition of IgG in mesentery may also be one of the important risk factors in the pathogenesis of renal lesions in HSPN [13]. Therefore, the duration of production, amount, and localization of IgA/ IgG circulating immune complexes may be the possible mechanisms of HSPN and responsible for the different presentation and symptoms in clinical.

In addition, cellular immune function disorder, especially helper $\mathrm{T}$ (Th) cell subsets disorder, plays a crucial role in HSPN [14]. Th1/Th2 imbalance is an important factor in immune response, Th cells differentiate into Th1 cells to trigger cell-mediated immunity responses and into Th2 cells to trigger the immunity and initiate allergic reactions, respectively [14]. An excessive Th2-dominated response has been characterized in children with HSP [15], which aggravates inflammatory response and promotes cytokines release, stimulating B cell to synthetize and secrete immunoglobulin [16]. The increase in Th2 immunodeviation in peripheral blood causes the development of vessel vasculitis and results in renal microvascular injury in patients with HSPN [14]. Consequently, Th1/Th2 imbalance may be another factor involved in the underlying pathogenesis mechanism of HSPN.

Accordingly, in this study, the therapeutic effects of QZXB GR on HSPN were evaluated; moreover, its potential mechanisms involved in the mitigation of $\operatorname{IgA}$ and $\operatorname{IgG}$ deposition and the regulation of Th1/Th2 immune imbalance were also investigated.

\section{Materials and Methods}

2.1. Drug Preparation. The eight ingredients of QZXB GR (151101, Beijing Shouer Pharmaceutical Factory) were Indigofera tinctoria L. (dried leaves and stems; $125 \mathrm{~g}$ ), Lithospermum erythrorhizon Siebold \& Zucc. (dried roots; $375 \mathrm{~g}$ ), Salvia miltiorrhiza Bunge (dried rhizome; $500 \mathrm{~g}$ ), Moutan officinalis (L.) Lindl. \& Paxton (dried root bark; $500 \mathrm{~g}$ ), Clematis chinensis Osbeck (dried rhizome; $375 \mathrm{~g}$ ), Agrimonia pilosa Ledeb. (dried aerial parts; 500 g), Kochia scoparia (L.) Schrad. (dried ripe fruit; $250 \mathrm{~g}$ ), and Dictamnus dasycarpus Turcz. (dried root bark; $250 \mathrm{~g}$ ). Briefly, Moutan officinalis (L.) Lindl. \& Paxton were distilled in water $(1: 16, \mathrm{w} / \mathrm{v})$ to extract paeonol, followed by the collection of the resulting distilled Liquid 1 and Herb Residues 1. Inclusion complex of paeonol with $\beta$-cyclodextrin was prepared. Indigofera tinctoria L., Lithospermum erythrorhizon Siebold \& Zucc., and Salvia miltiorrhiza Bunge were dipped $\left(40^{\circ} \mathrm{C}\right)$ extracting 2 times in $95 \%$ ethanol (1:10 and 1:8, w/v) for 24 hours, respectively, filtered, and concentrated (liquid : medicinal material ratio of $1: 1.00-1.05)$, followed by the collection of the resulting Extraction 1 and Herb Residues 2. Clematis chinensis Osbeck, Agrimonia pilosa Ledeb., Kochia scoparia (L.) Schrad., and Dictamnus dasycarpus Turcz. were added to the Herb Residues 1 and 2. They were cooked twice with 11 and 9 times the volume of water for $30 \mathrm{~min}$, respectively, filtered, combined with Liquid 1, and concentrated (liquid: medicinal material ratio of $1: 1.20-1.25)$ to obtain Extraction 2. Extraction 2, mannitol, dextrin, aspartame, and $\beta$-cyclodextrin inclusion complex of paeonol were mixed at a ratio of $1: 0.2: 0.4: 0.005: 1$ to prepare granules, dried, and sprayed with Extraction 1 below $60^{\circ} \mathrm{C}$. Finally, the mixture was sprayed $0.1 \%$ orange flavor and mixed to produce the QZXB GR formula.

The following drugs were used: Xuening capsule (XN Caps, 150803, Yantai Zhongzhou Pharmacy, China) and mycophenolate mofetil (MMF, SH0065, Shanghai Roche Pharmaceuticals, China).

2.2. Animals. Male and female KM mice ( $n=64$ per gender, 14-16 g, 3-4 weeks) were obtained from Vital River Experimental Animal Services (Beijing, China) and housed under pathogen-free conditions with controlled temperature of $24 \pm 2^{\circ} \mathrm{C}$ and humidity of $60 \pm 5 \%$ with a $12 \mathrm{~h}$ light/dark cycle. Because HSP most commonly occurs in children, young mice (14-16 g) were used in this study, and similar results were obtained using mice of different genders. Standard laboratory chow and water were provided ad libitum. All animal experiments were approved by the Experimental Animal Care and Use Committee of the Institute of Materia Medica, Chinese Academy of Medical Sciences \& Peking Union Medical College (No. 20160612). 
2.3. Gliadin plus Indian Ink-Induced HSPN in Mice and Treatment. Mice were randomly divided into 8 groups: control group, model group, QZXB GR $(3.6 \mathrm{~g} / \mathrm{kg} / \mathrm{day}, 6$ times of dose in clinical practice) group, QZXB GR $(7.2 \mathrm{~g} / \mathrm{kg} /$ day $)$ group, QZXB GR (14.4 g/kg/day) group, XN Caps $(0.84 \mathrm{~g} / \mathrm{kg} /$ day, 12 times of dose in clinical practice) group, $\operatorname{MMF}(0.3 \mathrm{~g} /$ $\mathrm{kg} /$ day, 12 times of dose in clinical practice) group, and QZXB GR $(7.2 \mathrm{~g} / \mathrm{kg} /$ day $)+\mathrm{MMF}(0.3 \mathrm{~g} / \mathrm{kg} /$ day $)$ group. All groups except control received tail-vein injection of Indian Ink (Solarbio, China) $0.5 \mathrm{mg} / 10 \mathrm{~g}$ once a week for 3 weeks, followed by intragastric administration of $0.5 \mathrm{ml}$ of $1 \mathrm{mg} / \mathrm{ml}$ Gliadin (Sigma-Aldrich, USA) in $6 \mathrm{mmol} / \mathrm{l} \mathrm{HCl}$ every two days for 11 weeks, and finally, tail-vein injection of the mixture of $0.1 \mathrm{ml}$ PBS and $0.1 \mathrm{ml}$ of freshly prepared $10 \mathrm{mg} / \mathrm{ml}$ Gliadin every day for three days. Control group received vehicle. The detail of experimental design is shown in Figure 1(a).

All groups except control and model administrated intragastrically corresponding drugs at different doses every day during 9-16 week. Control and model groups received equal amount of saline. At the end of $16^{\text {th }}$ week, mice were sacrificed via euthanasia. Skin, urine, blood, and kidney samples were collected for further analysis.

2.4. Histopathologic Assessment of Kidney Tissues. Kidneys were immersion-fixed in $4 \%$ paraformaldehyde, dehydrated, paraffin embedded, and sectioned at $4 \mu \mathrm{m}$ for hematoxylin and eosin (H\&E) staining and periodic acid-Schiff base (PAS) staining. The glomerular damage and renal tubular injury were evaluated, respectively, in H\&E stain as follows: (1) glomerular damage, such as glomerular interstitial (mesangial) proliferation, renal capsule cavity shrinkage or disappearance, glomus atrophy, and basement membrane thickening; (2) renal tubular injury, such as vacuolar degeneration of renal tubular epithelial cells, tubular atrophy, and cast. A pathological score was given according to the injury ratio of glomerulus or renal tubule: $0=$ no injury; $1=1 \%-25 \%$ injury ratio; $2=26 \%-50 \%$ injury ratio; and $3=51 \%-100 \%$ injury ratio. The renal interstitial inflammation in H\&E stain was scored as follows: $0=$ normal, $1=$ slight, 2 =moderate, and $3=$ severe. Besides, mean diameter and optical density of glomerular were digitally quantified in PAS stain.

2.5. Subcutaneous Hemorrhage Evaluation. The skin of left hindlimb (about $1.5 \mathrm{~cm} \times 1.5 \mathrm{~cm}$ ) was obtained and pictured for subcutaneous hemorrhage score according to the following criteria: $0=$ no subcutaneous capillaries, petechiae, or ecchymosis; 1 = visible capillaries but no obvious telangiectasia; 2 = two or more sites with obvious telangiectasia; and $3=$ visible subcutaneous hemorrhage and ecchymosis.

2.6. Urine Sample Collection and Analysis. Fresh urine microscopy for RBC, WBC, and CAST was conducted every two weeks during weeks 9-16. The 24-hour urine was collected using metabolic cages before scarification to examine the volume of urine and $24 \mathrm{~h}$ urinary protein excretion with BCA protein assay kit (Solarbio, China).
2.7. Serum Sample Collection and Analysis. Blood samples were collected from the eyeball of mice, clotted, and centrifuged at $1,000 \mathrm{~g}$ for $20 \mathrm{~min}$ at $20^{\circ} \mathrm{C}$. Levels of interleukin-6 (IL-6) and circular immune complex (CIC) were assessed by mouse IL-6 enzyme-linked immunosorbent assay (ELISA) kit (Biolegend, USA) and mouse CIC ELISA kit (Senbeijia Biological Technology, China), respectively. Total protein (TP), albumin (ALB), creatinine (Cre), and blood urea nitrogen (BUN) in serum were evaluated by blood biochemical analyzer (TOSHIBA, Japan) with commercial kits (Biosino Biotechnology and Science, China) according to the manufacturer's protocols.

2.8. Immunohistochemistry Studies of $\operatorname{IgA}$ and $\operatorname{IgG}$ in Kidney Tissues. The paraffin-embedded kidney sections $(5 \mu \mathrm{m})$ were rehydrated, antigens retrieved using heated citrate, incubated with rabbit antimouse IgA and IgG (Bethyl Laboratories, USA), and visualized using horseradish peroxidase(HRP-) coupled secondary antibodies (Cell Signaling Technology, USA). The positive staining of mesangial, vascular loop, tubular basement membrane (TBM), and arteriole wall was evaluated by integrated optical density, and a score was given as follows: $0=$ no positive staining cells; $1=1 \%-25 \%$ positive staining; $2=26 \%-50 \%$ positive staining; and $3=51 \%-100 \%$ positive staining.

2.9. Flow Cytometrical Analysis. A single-cell suspension was prepared from blood sample or spleen sample. Blood cells were incubated with anti-CD3-PE and anti-CD19-PE-Cy7 monoclonal antibody (BD Biosciences, USA) at $4^{\circ} \mathrm{C}$ for $30 \mathrm{~min}$. Spleen cells were incubated with anti-CD3-PE, antiCD4-FITC, and anti-CD8-Percp-Cy5.5 monoclonal antibody (BD Biosciences, USA) at $4^{\circ} \mathrm{C}$ for $30 \mathrm{~min}$. For intracellular staining, cells were fixed and permeabilized after surface staining, and anti-IL-4-APC and anti-IFN- $\gamma$-PE-Cy7 (eBioscience, USA) were then added to stain Th2 cells and Th1 cells. Stained cells were analyzed by flow cytometric analysis using a flow cytometer (ACEA NovoCyte 2060R, China).

2.10. Statistical Analysis. Data were expressed as mean\pm standard error (SEM). As the normality test by Kolmogorov-Smirnov test (K-S test) was passed, data were analyzed using Student's $t$-test for comparison between two groups and one-way ANOVA for multiple groups followed by Fisher's least significant difference (LSD) test, otherwise, using Mann-Whitney $U$ test. Values of $P<0.05$ were regarded as significant. The above analyses were conducted with GraphPad Prim 6.0 and SPSS 19.0 statistical software.

\section{Results}

3.1. QZXB GR Attenuated Renal Pathological Damages in HSPN Mice. Hematoxylin-eosin (H\&E) and periodic acidSchiff (PAS) staining were used to assess the renal histopathologic changes in HSPN mice. A pathological score was used to quantify the glomerular injury, renal tubal injury, 


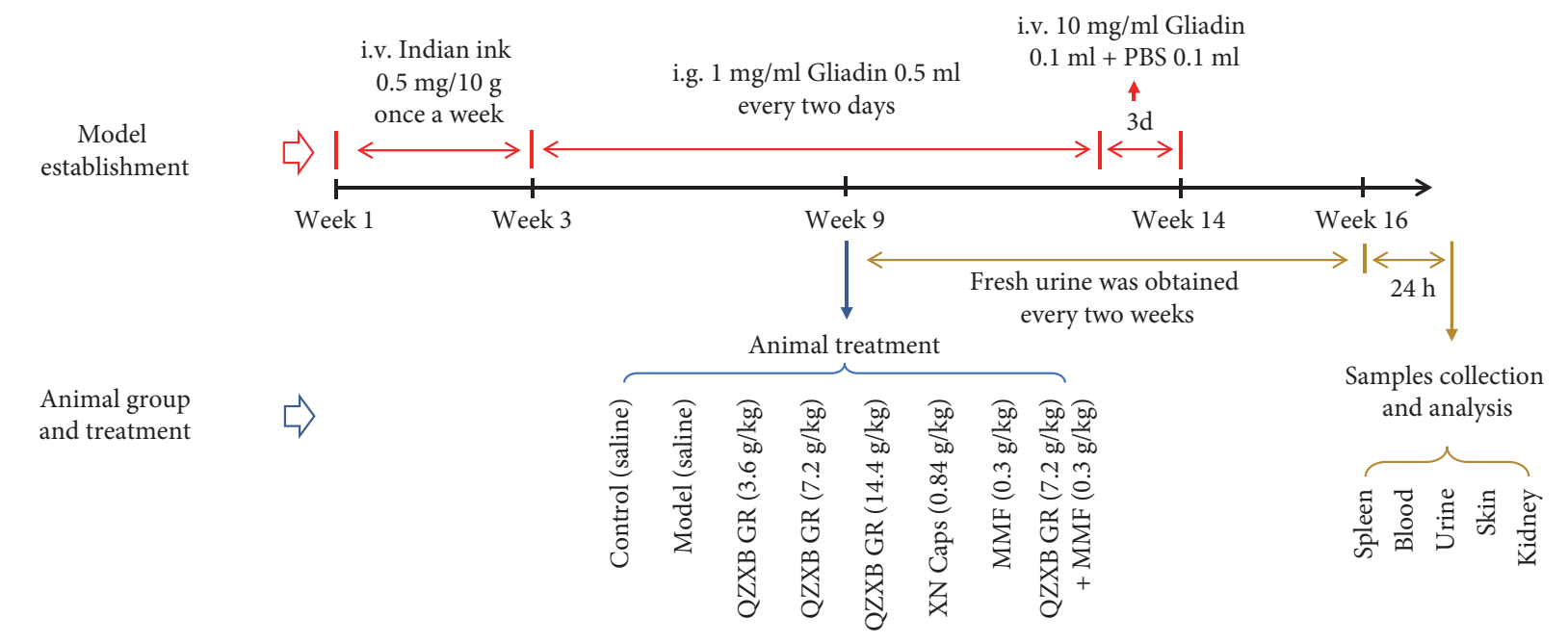

(a)

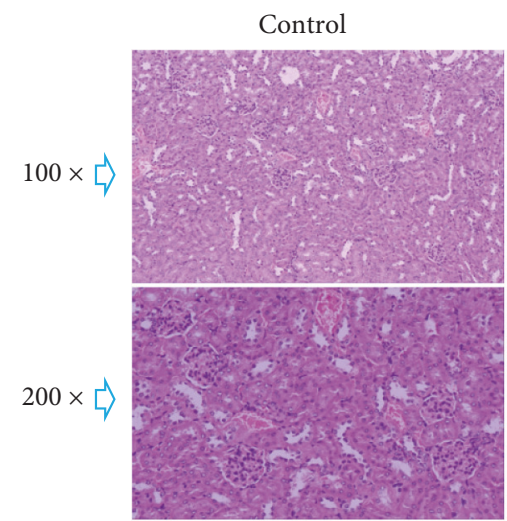

QZXB GR (14.4 g/kg)

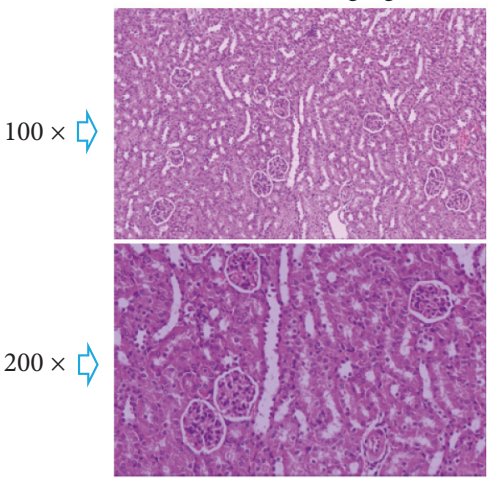

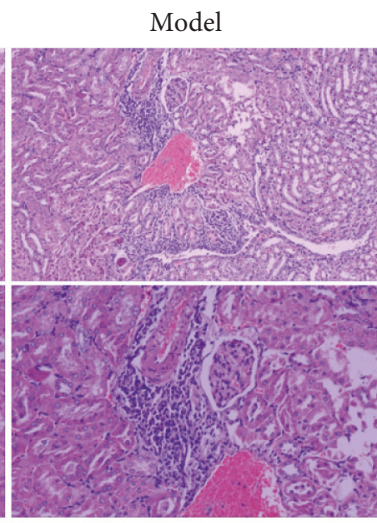

XN Caps
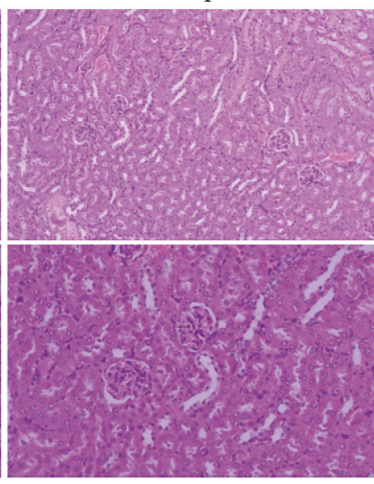

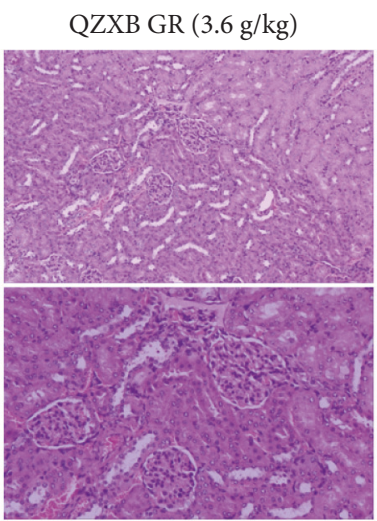

MMF

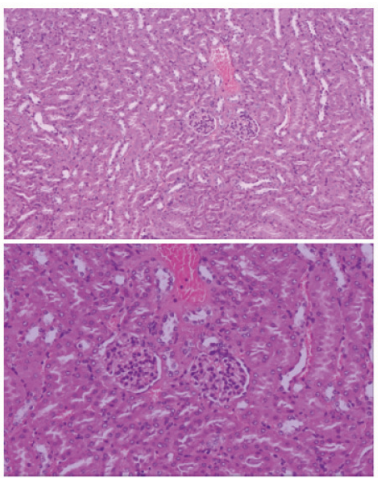

QZXB GR $(7.2 \mathrm{~g} / \mathrm{kg})$

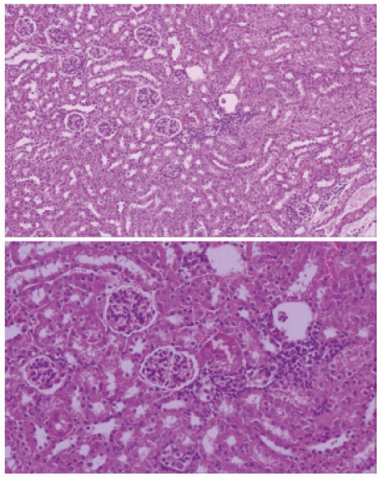

QZXB GR (7.2 g/kg) + MMF

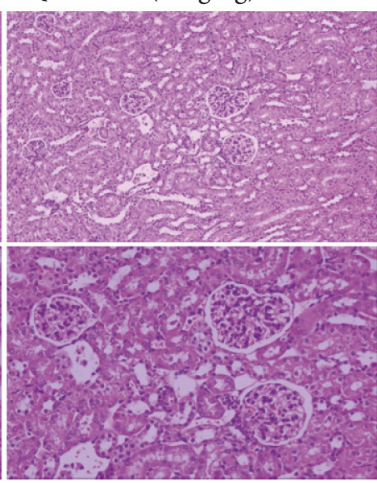

(b)

Figure 1: Continued. 


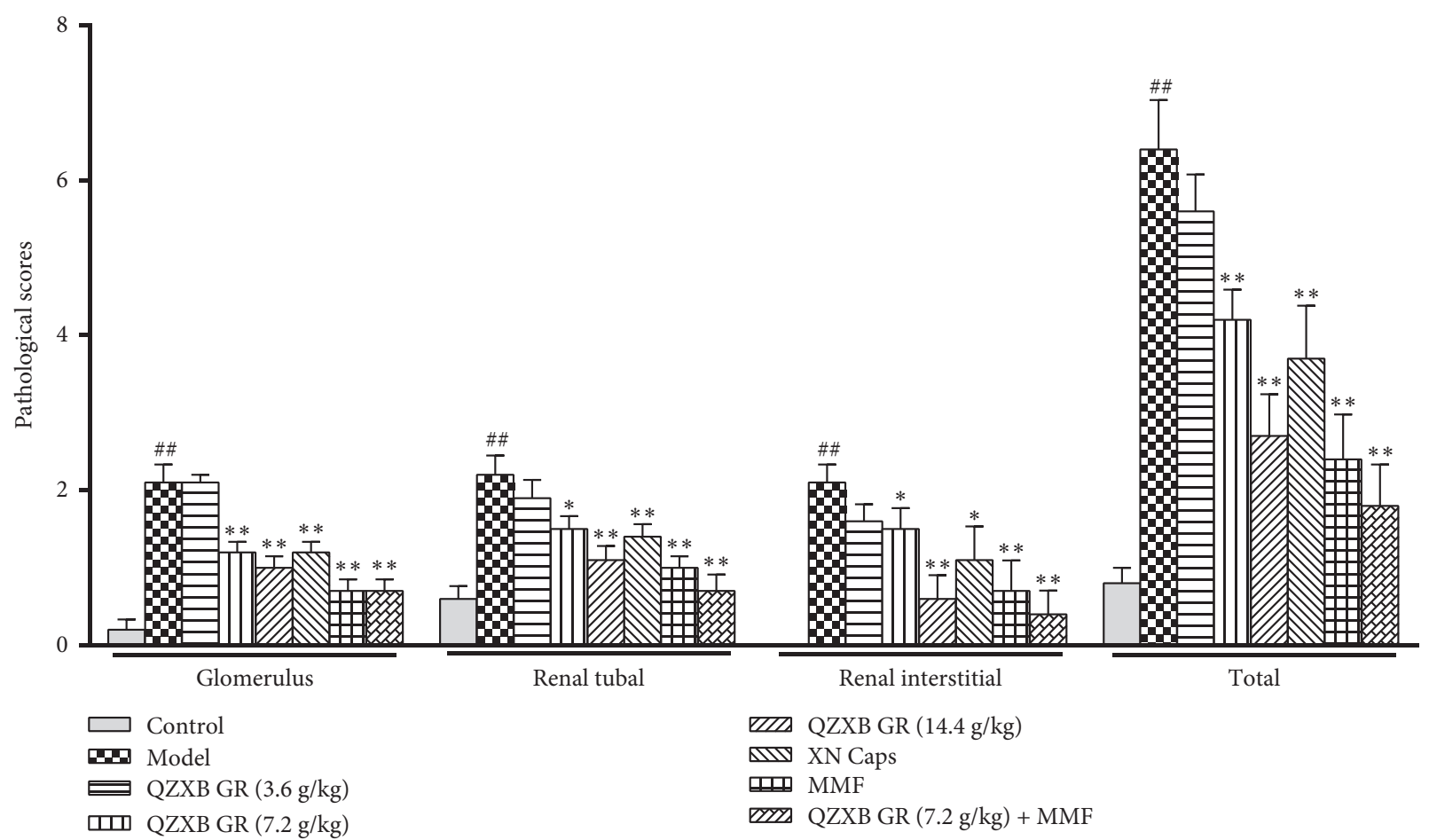

(c)

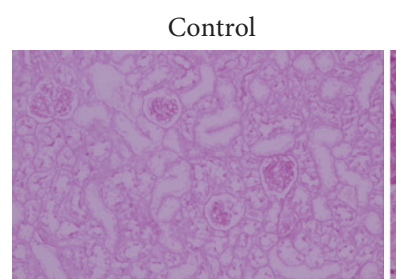

QZXB GR (14.4 g/kg)

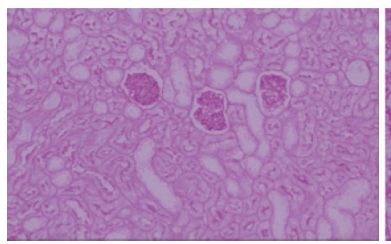

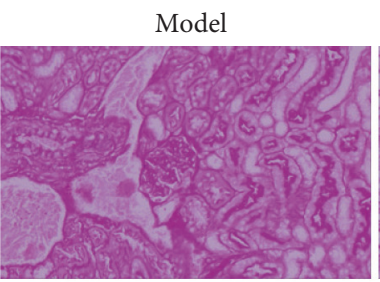

XN Caps

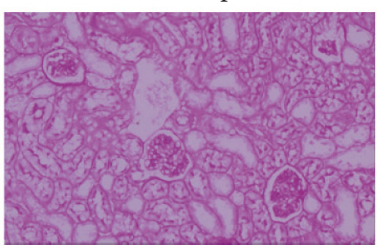

(d)

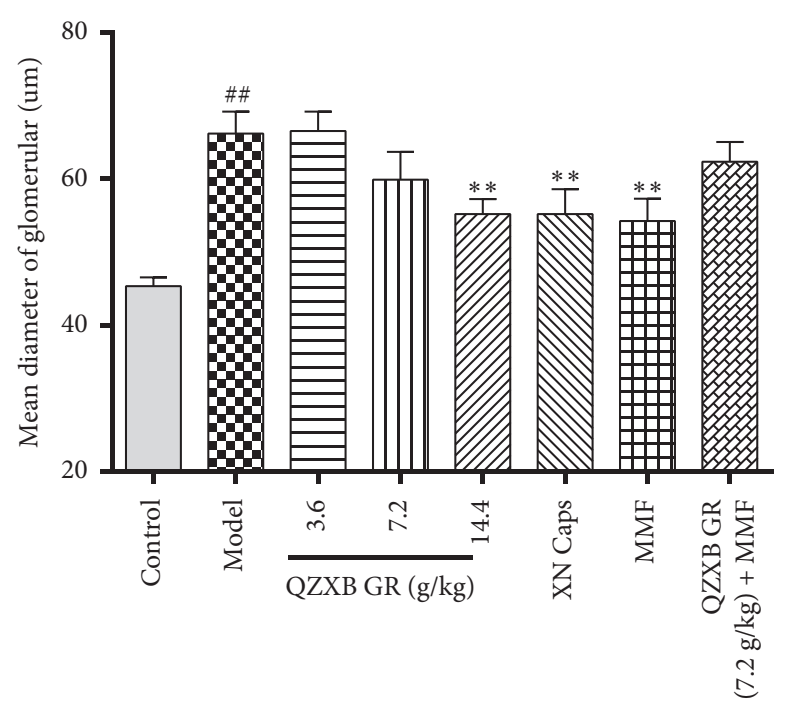

(e)

Figure 1: Continued.

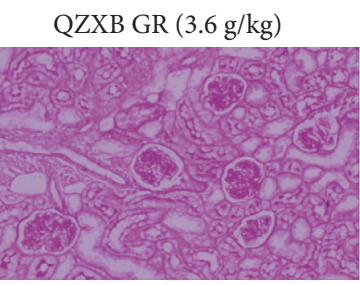

MMF

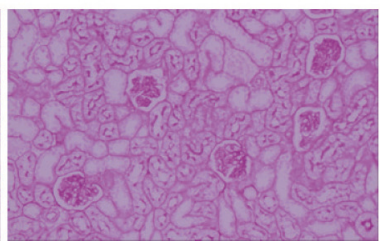

QZXB GR $(7.2 \mathrm{~g} / \mathrm{kg})+\mathrm{MMF}$
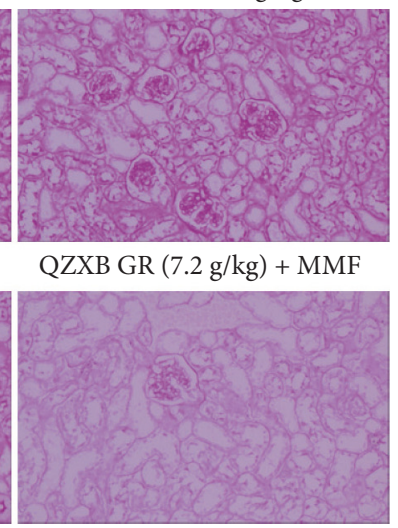


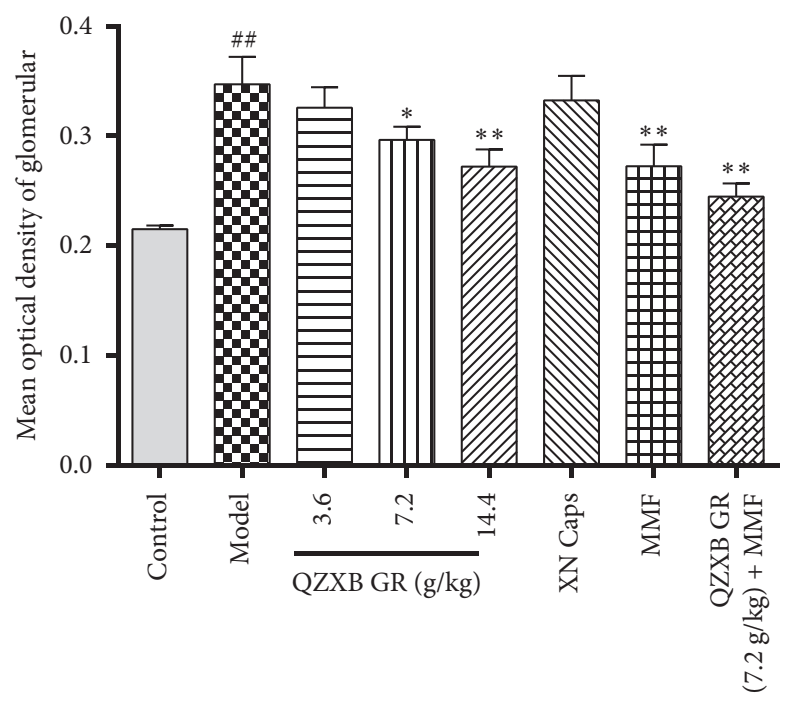

(f)

FIgURE 1: QZXB GR attenuated renal pathological damages in HSPN mice. (a) Timeline for the development and treatment process of HSPN. (b) Images of pathological changes in renal tissues stained by H\&E staining (magnified $\times 100$ and $\times 200$ ). (c) Quantification of pathological scoring of renal injury in H\&E staining. (d) Images of pathological changes in renal tissues with PAS staining (magnified $\times 200$ ). (e) Quantification of glomerular diameters in PAS-stained renal sections. (f) Quantification of optical density in PAS-stained renal sections. Data were presented as means \pm SEM, $n=10$. ${ }^{\#} P<0.05$ and ${ }^{\# \#} P<0.01$ versus control group, ${ }^{*} P<0.05$ and ${ }^{* *} P<0.01$ versus HSPN model group.

and renal interstitial inflammation in H\&E staining. As shown in Figures 1(b) and 1(c), QZXB GR (7.2 g/kg and $14.4 \mathrm{~g} / \mathrm{kg}$ ) treatment significantly reduced the pathological scores of glomerulus, renal tubal, and renal interstitial injury compared with the model group $(P<0.05$ or 0.01$)$, as same as positive control XN Caps and MMF treatment. Furthermore, QZXB GR $(7.2 \mathrm{~g} / \mathrm{kg})$ combined with $\mathrm{MMF}$ treatment behaved more effective in renal tubal, renal interstitial injury, and total pathological scores compared with QZXB GR and MMF single treatment.

PAS staining was used to observe the pathological changes of glomerular basement membrane. As shown in Figures 1(d) and 1(e), compared with the model group, QZXB GR $(14.4 \mathrm{~g} / \mathrm{kg})$, XN Caps, and MMF treatment significantly decreased HSPN mice glomerular diameter $(P<0.01)$. Additionally, QZXB GR $(7.2$ and $14.4 \mathrm{~g} / \mathrm{kg})$ treatment also notably reduced glomerular mean optical density in HSPN mice $(P<0.05$ or 0.01$)$, as well as that of $\mathrm{MMF}$ and QZXB GR $(7.2 \mathrm{~g} / \mathrm{kg})+\mathrm{MMF}$ treatment $(P<0.01)$. These results showed potential protective effects of QZXB GR on renal tissue pathological damages in HSPN mice.

3.2. QZXB GR Reduced Subcutaneous Hemorrhage, Proteinuria, and Hematuria in HSPN Mice. As shown in Figures 2(a) and 2(b), the subcutaneous hemorrhage on left legs was pronounced in HSPN model mice, which might be significantly decreased with QZXB GR treatment $(P<0.01)$, as well as XN Caps and MMF treatment. Furthermore, the subcutaneous hemorrhage was collaboratively inhibited by combination treatment of QZXB GR $(7.2 \mathrm{~g} / \mathrm{kg})$ and MMF.
Then, 24-hour urine was collected, and its volume and protein excretion were determined. As shown in Figure 2(c), there were no significant changes in urine volume among all experimental groups $(P>0.05)$. However, compared with the control mice, $24 \mathrm{~h}$ urinary protein levels were significantly increased in HSPN model mice $(P<0.01)$, which might be significantly reduced with QZXB GR $(14.4 \mathrm{~g} / \mathrm{kg})$ treatment $(P<0.05)$, as well as XN Caps, MMF, and QZXB GR $(7.2 \mathrm{~g} / \mathrm{kg})+\mathrm{MMF}$ treatment $(P<0.05$ or 0.01 ; Figure 2(d)).

From $9^{\text {th }}$ week, the numbers of RBC (Figure $2(\mathrm{e})$ ), WBC (Figure 2(f)), and CAST (Figure 2(g)) in fresh urine were counted by microscopy every two weeks. Compared with the control group, the counts of urinary RBC, WBC, and CAST were significantly increased in the HSPN model group $(P<0.01)$. QZXB GR treatment might significantly decrease urinary $\mathrm{RBC}$ and $\mathrm{WBC}$ count after $13^{\text {th }}$ week $(P<0.05$ or 0.01 ), as well as XN Caps treatment, whereas MMF treatment exerted the effect after $14^{\text {th }}$ week. The effects were facilitated by combinatory use of QZXB GR with MMF.

3.3. QZXB GR Decreased the Levels of Serum CIC, IL-6, and Regulated Renal Biochemical Parameters in HSPN Mice. The serum CIC and IL- 6 were determined by ELISA. As shown in Figure 3(a), compared with the model group, QZXB GR $(14.4 \mathrm{~g} / \mathrm{kg})$ treatment significantly decreased the serum CIC $(P<0.05)$, similar to that of $\mathrm{MMF}$, and a superimposed effect might be observed in combination treated group. As shown in Figure 3(b), QZXB GR treatment could also significantly inhibit the levels of serum IL-6 in HSPN mice $(P<0.01)$, as well as XN Caps, MMF, and QZXB GR $(7.2 \mathrm{~g} / \mathrm{kg})+\mathrm{MMF}$ treatment. 

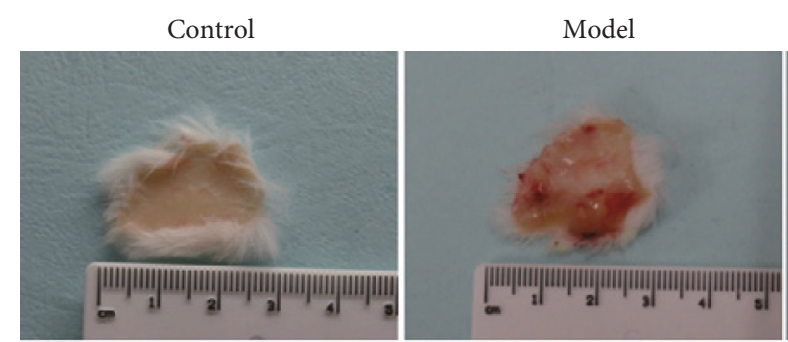

QZXB GR (3.6 g/kg)

QZXB GR $(7.2 \mathrm{~g} / \mathrm{kg})$

QZXB GR $(14.4 \mathrm{~g} / \mathrm{kg})$

XN Caps
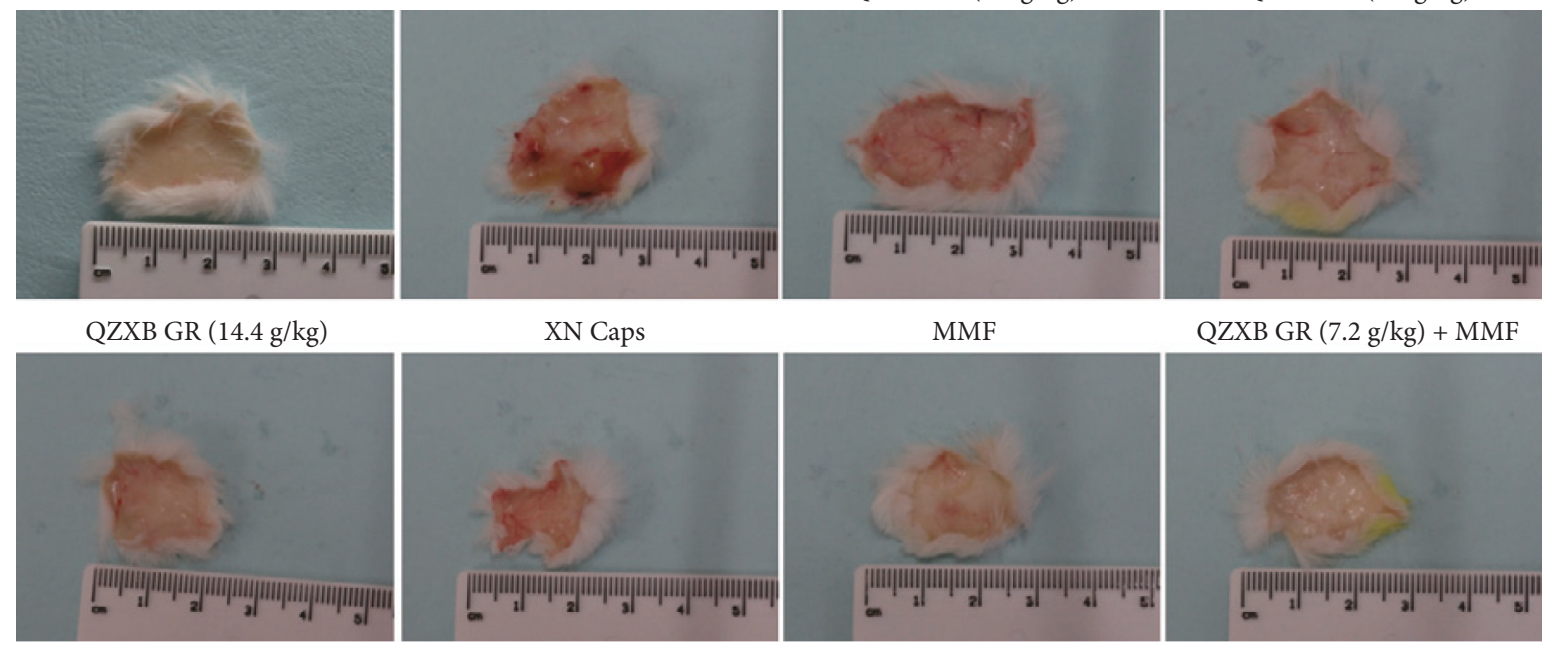

MMF

QZXB GR $(7.2 \mathrm{~g} / \mathrm{kg})+\mathrm{MMF}$
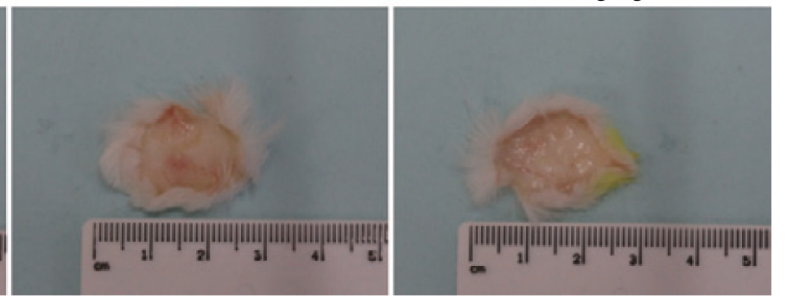

(a)

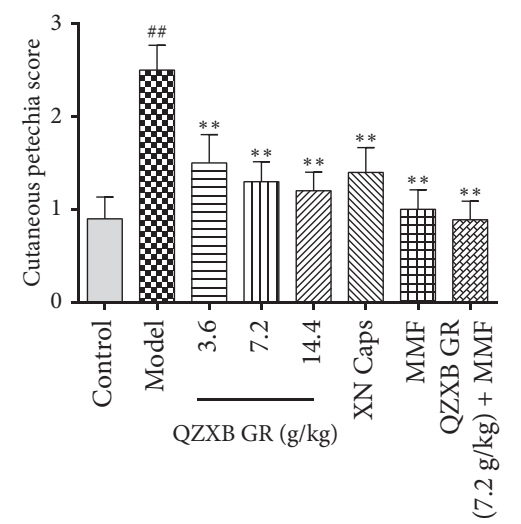

(b)

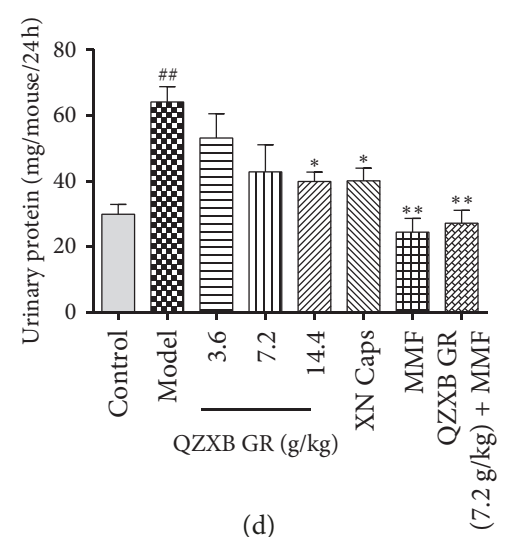

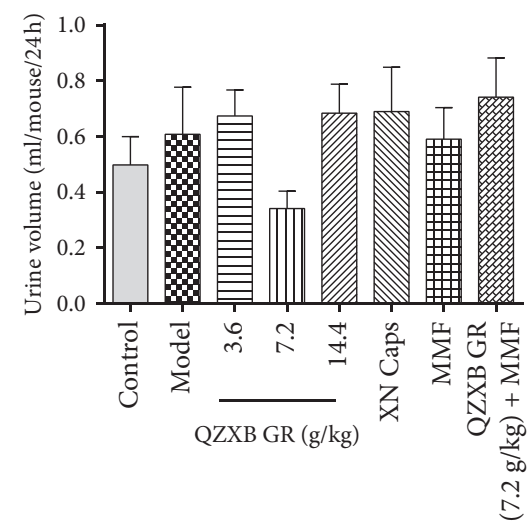

(c)

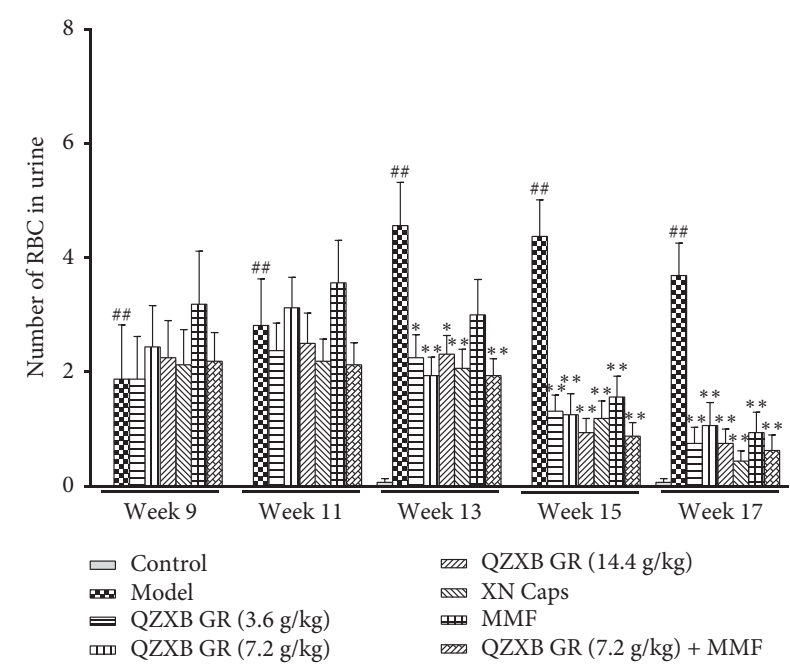

(e)

FIgure 2: Continued. 


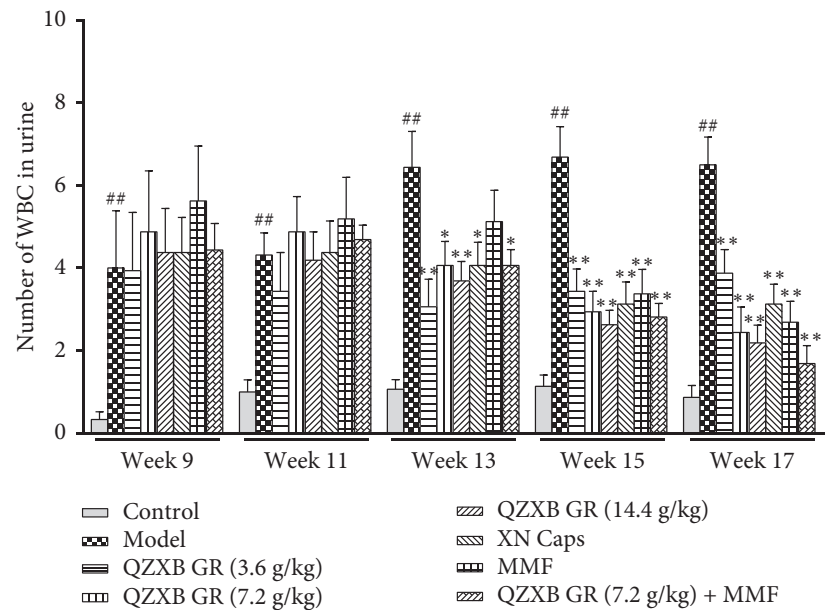

(f)

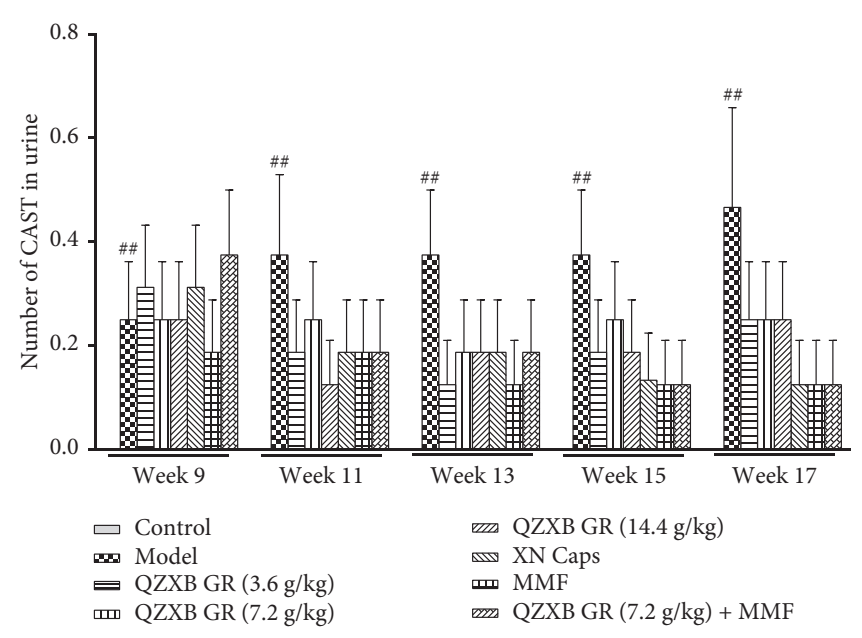

(g)

FIGURE 2: QZXB GR reduced subcutaneous hemorrhage, hematuria, and proteinuria in HSPN mice. (a) Images of subcutaneous hemorrhage in HSPN mice $(n=10)$. (b) Quantitative scoring of subcutaneous hemorrhage in HSPN mice $(n=10)$. (c) 24-hour urine volume after the last treatment $(n=8)$. (d) 24-hour urinary protein excretion after the last treatment $(n=8)$. (e) Numbers of RBC in fresh urine of HSPN mice from $9^{\text {th }}$ week $(n=16)$. (f) Numbers of WBC in fresh urine of HSPN mice from $9^{\text {th }}$ week $(n=16)$. (g) Numbers of CAST in fresh urine of HSPN mice from $9^{\text {th }}$ week $(n=16)$. Data were presented as means \pm SEM. ${ }^{\#} P<0.05$ and ${ }^{\# \#} P<0.01$ versus control group; ${ }^{*} P<0.05$ and ${ }^{* *} P<0.01$ versus HSPN model group.

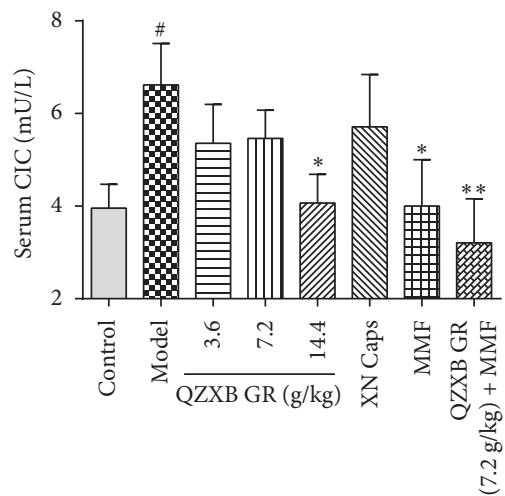

(a)

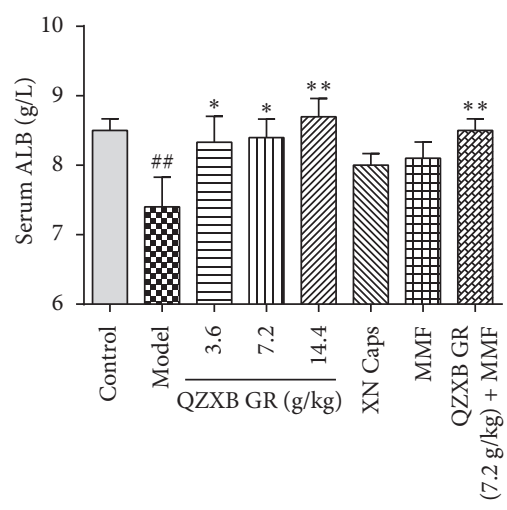

(d)

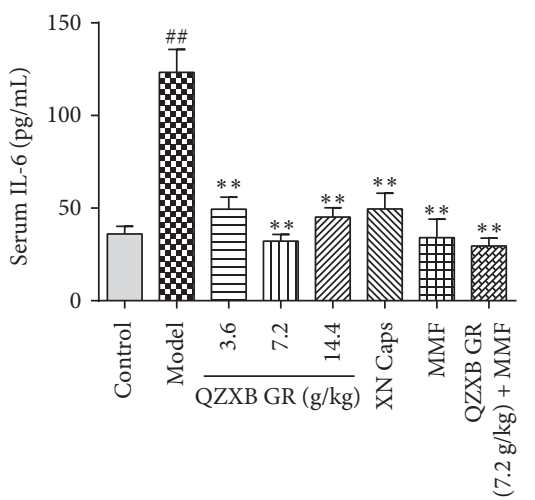

(b)

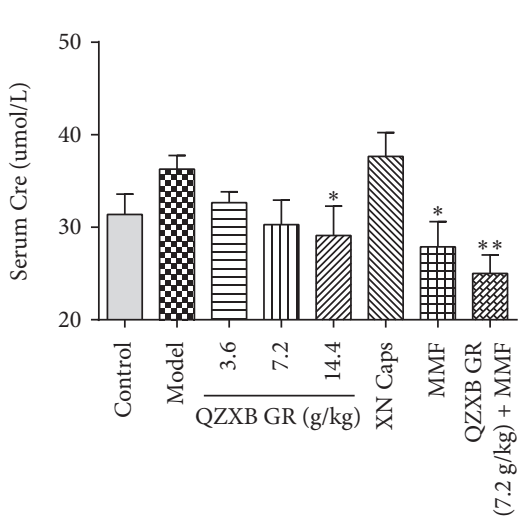

(e)

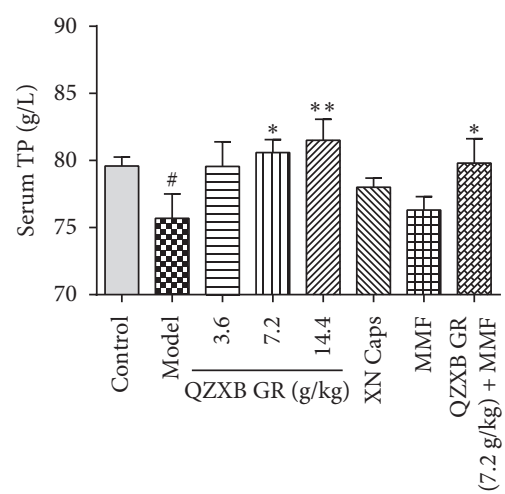

(c)

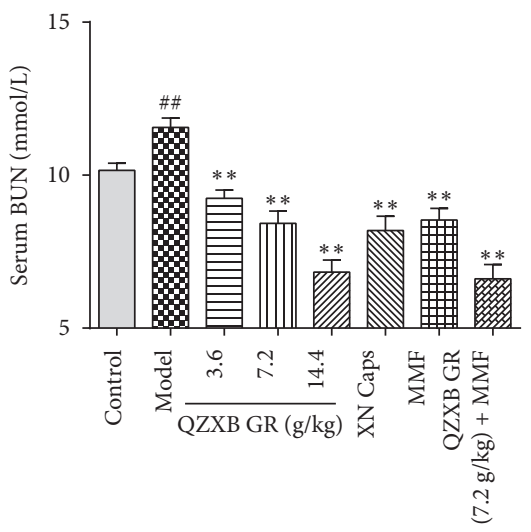

(f)

Figure 3: Continued. 


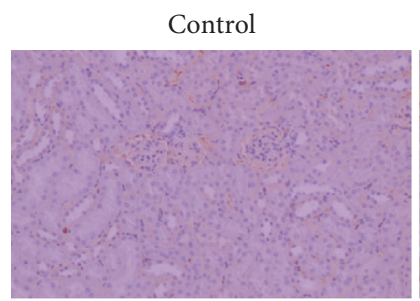

QZXB GR (14.4 g/kg)

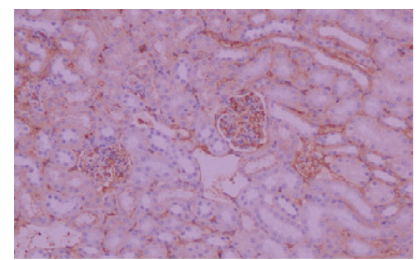

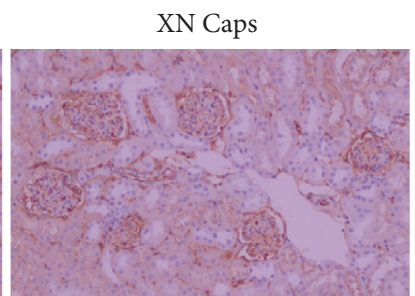

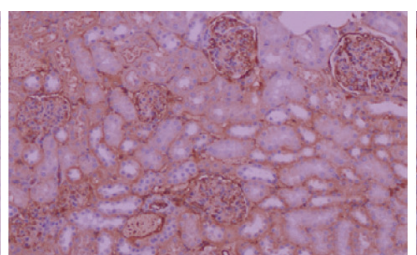

XN Caps

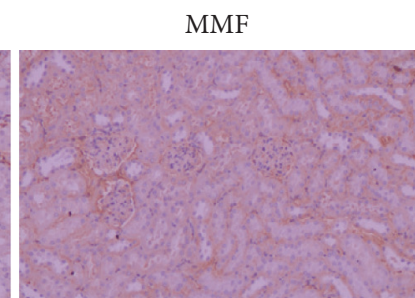

QZXB GR (3.6 g/kg)

QZXB GR $(7.2 \mathrm{~g} / \mathrm{kg})$
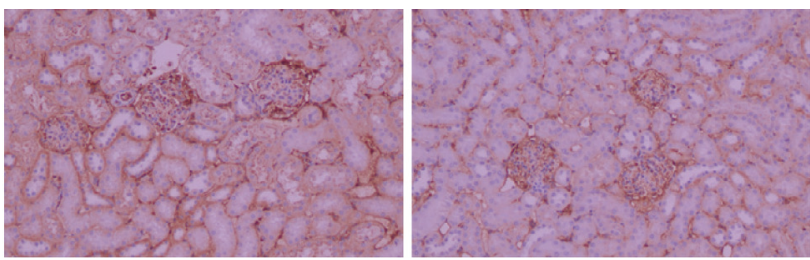

QZXB GR $(7.2 \mathrm{~g} / \mathrm{kg})+\mathrm{MMF}$

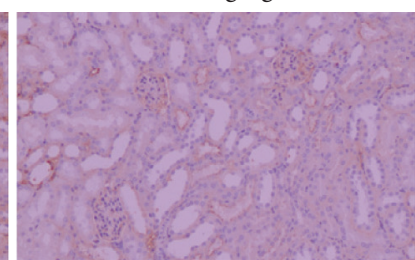

(g)
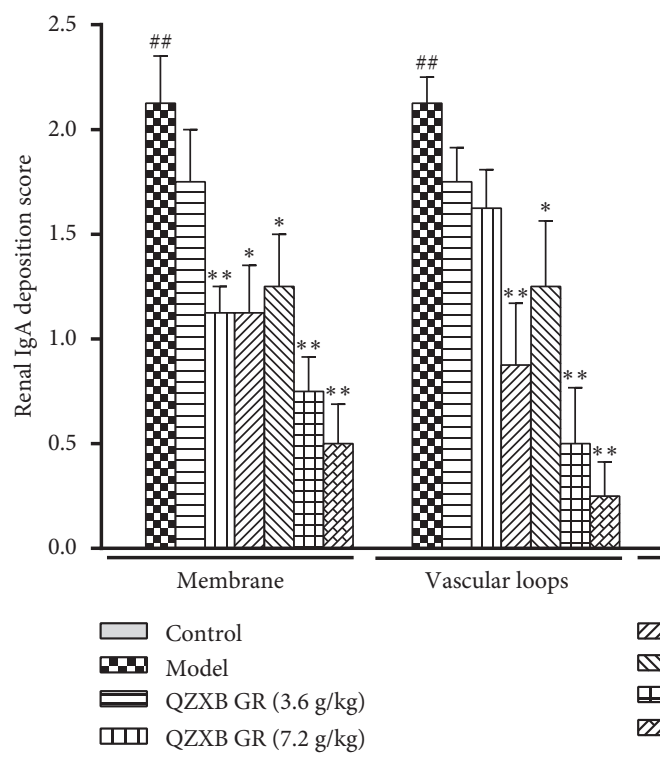

Vascular loops

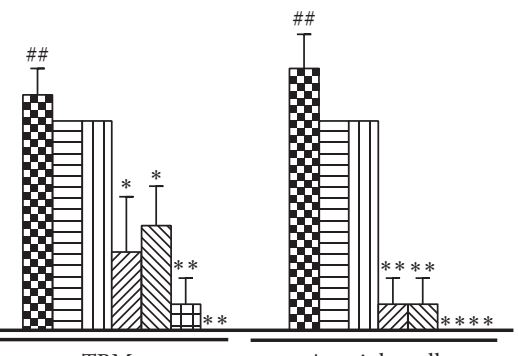

TBM

Arteriole wall

ש2] QZXB GR $(14.4 \mathrm{~g} / \mathrm{kg})$

XN Caps

MMF

[27] QZXB GR $(7.2 \mathrm{~g} / \mathrm{kg})+\mathrm{MMF}$

(h)

Control

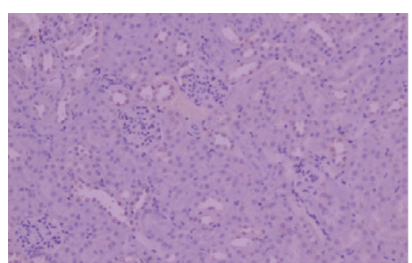

QZXB GR (14.4 g/kg)

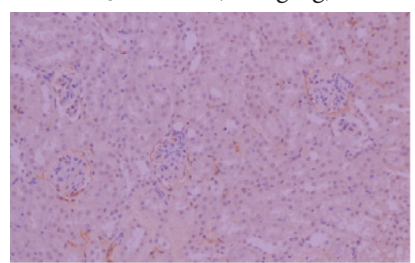

Model

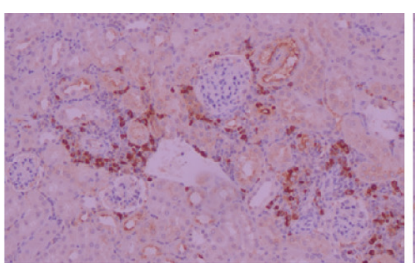

XN Caps

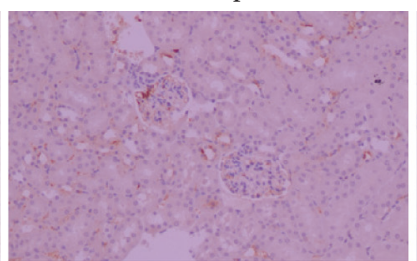

QZXB GR (3.6 g/kg)

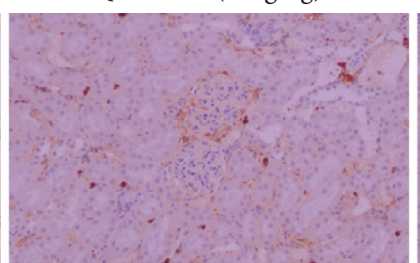

MMF

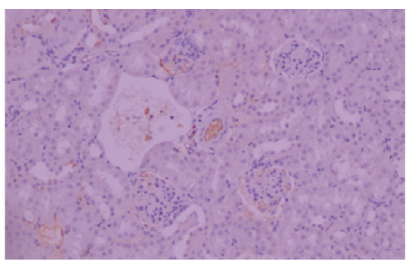

(i)
QZXB GR (7.2 g/kg)

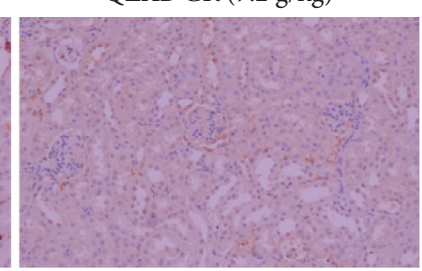

QZXB GR (7.2 g/kg) + MMF

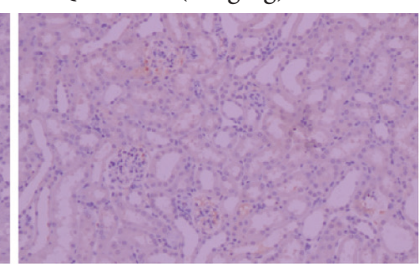

FIgURE 3: Continued. 


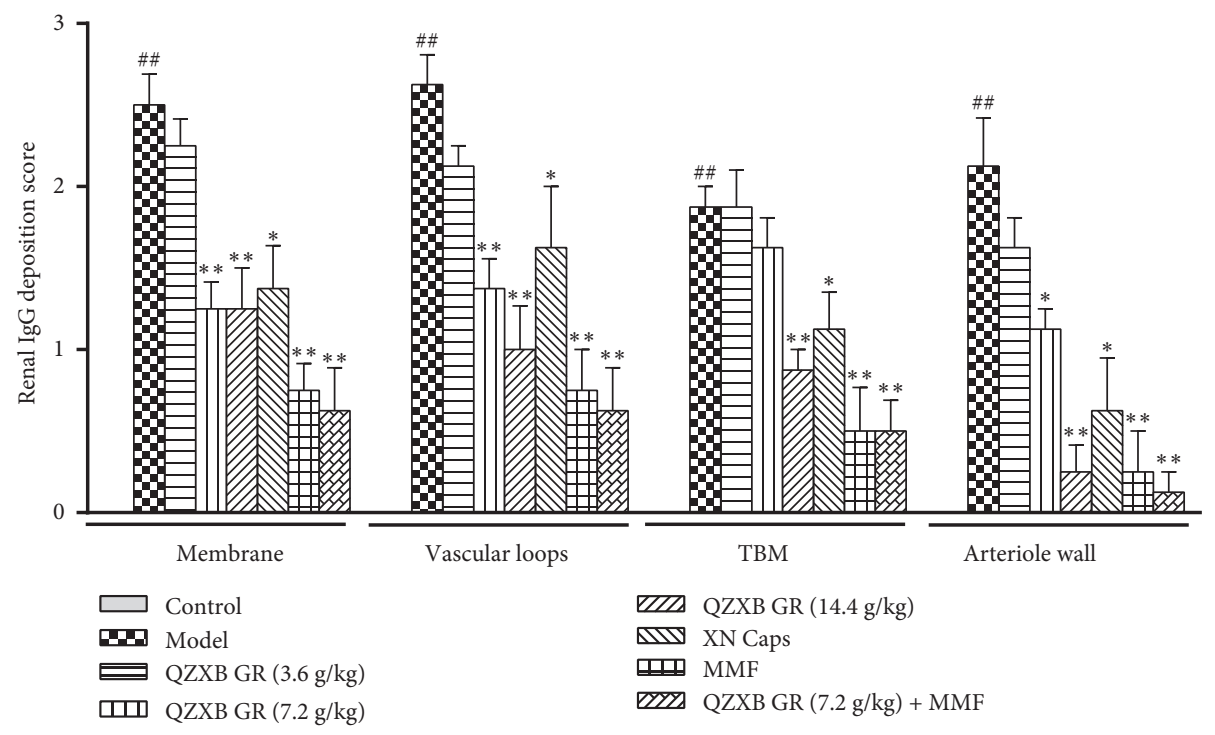

(j)

Figure 3: QZXB GR decreased the levels of CIC, IL-6, renal biochemical parameters in the serum and suppressed immune complex deposition in HSPN mice kidneys. (a) The level of serum CIC in HSPN mice. (b) The level of serum IL-6 in HSPN mice. (c) The level of serum TP in HSPN mice. (d) The level of serum ALB in HSPN mice. (e) The level of serum Cre in HSPN mice. (f) The level of serum BUN in HSPN mice. (g) Representative photomicrographs of IgA deposition $(\times 200)$. (h) Quantitative scoring of IgA deposition. (i) Representative photomicrographs of IgG deposition $(\times 200)$. (j) Quantitative scoring of IgG deposition. Data were presented as means \pm SEM, $n=8$. ${ }^{\#} P<0.05$ and ${ }^{\# \#} P<0.01$ versus control group, ${ }^{*} P<0.05$ and ${ }^{* *} P<0.01$ versus $\mathrm{HSPN}$ model group.

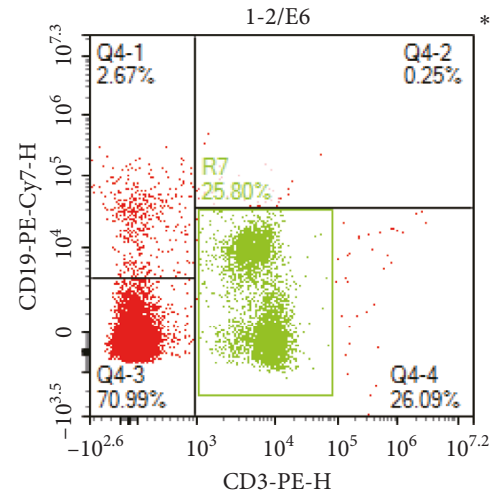

(A)

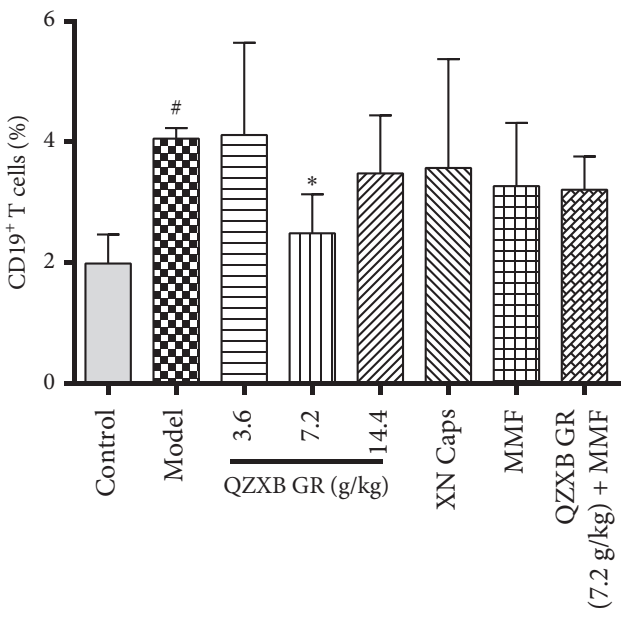

(B)

(a)

Figure 4: Continued. 


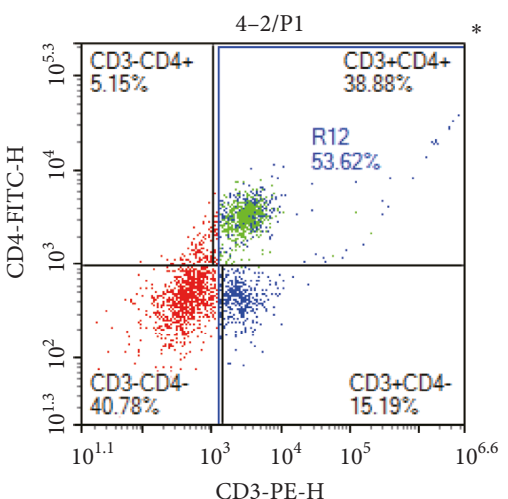

(A)

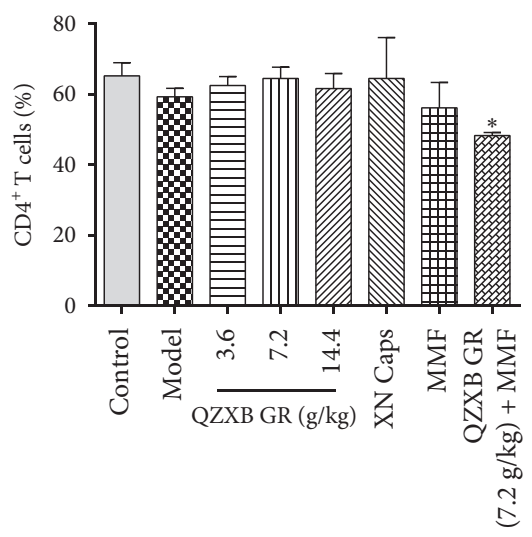

(D)

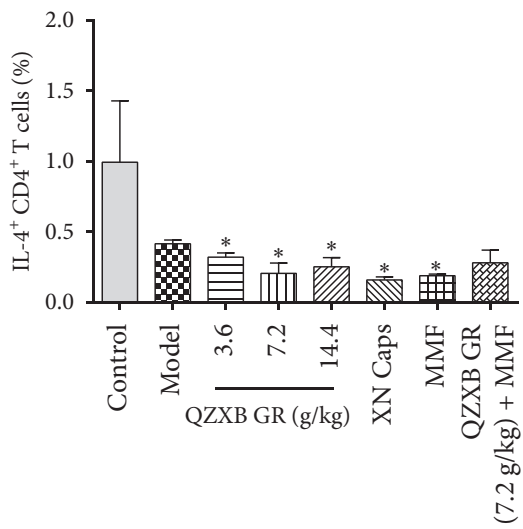

(G)

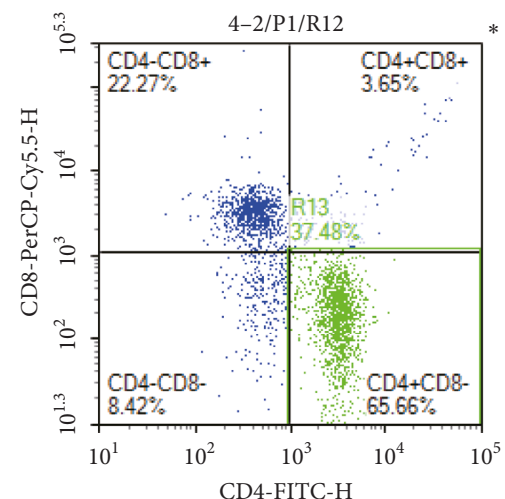

(B)

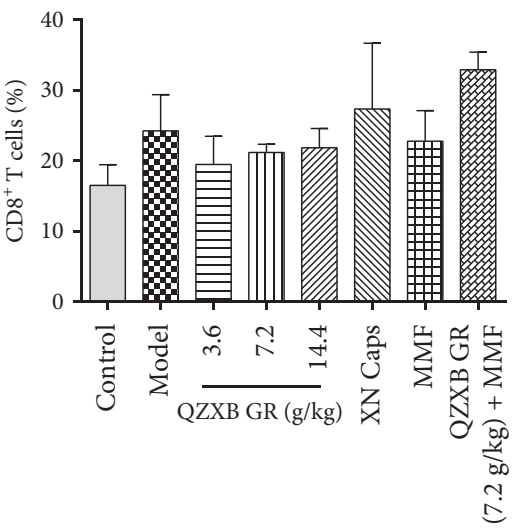

(E)

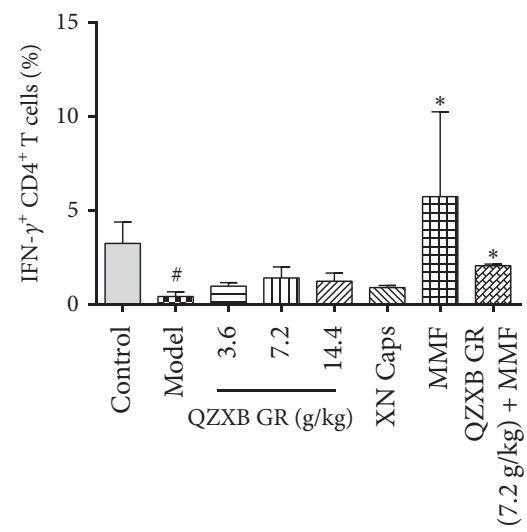

(H)

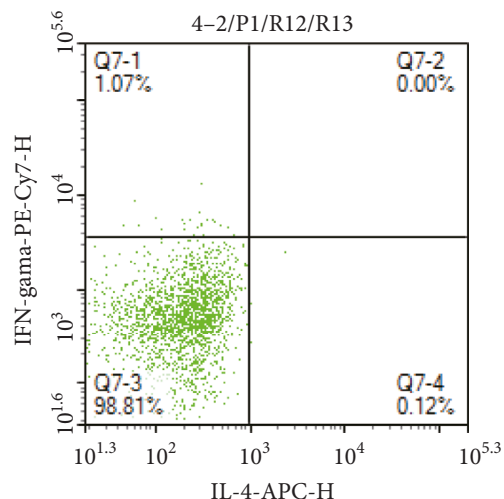

(C)

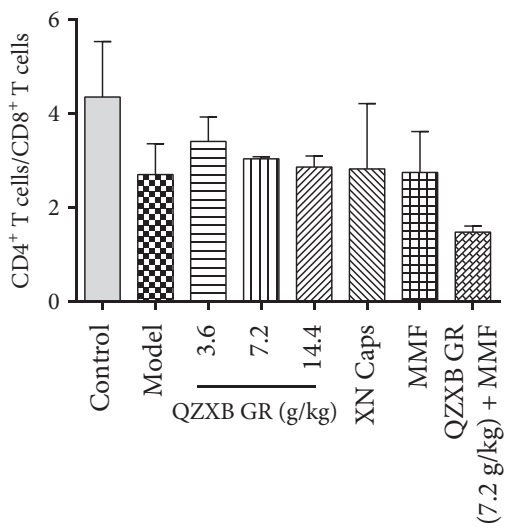

(F)

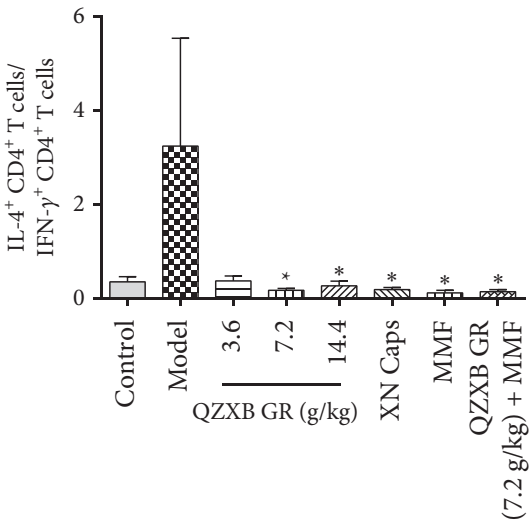

(I)

(b)

FIGURE 4: Effects of QZXB GR on lymphocyte subsets in HSPN mice. (a) The lymphocyte subsets in the peripheral blood. (A) Lymphocytes were stained with anti-CD3 and anti-CD19. (B) Quantification of CD19+ B cells. (b) The lymphocyte subsets in the spleen lymphocytes. (A-C) The gating strategy. (D) The percentage of CD4+ T cells. (E) The percentage of CD8+ T cells. (F) The ratio of CD4+ T cells/CD8+ T cells. (G) The percentage of CD4+ IL-4+ T cells. (H) The percentage of CD4+ IFN- $\gamma+$ T cells, and (I) the ratio of CD4+ IL-4+ T cells/CD4+ IFN- $\gamma+$ T cells were shown. Data were presented as means \pm SEM, $n=3$. ${ }^{\#} P<0.05$ and ${ }^{\# \#} P<0.01$ versus control group; ${ }^{*} P<0.05$ and ${ }^{* *} P<0.01$ versus HSPN model group.

Furthermore, the renal biochemical parameters, including serum TP (Figure 3(c)), ALB (Figure 3(d)), Cre (Figure 3(e)), and BUN (Figure 3(f)), were measured by blood biochemical analyzer. Compared with the control mice, the serum TP and ALB were significantly decreased, whereas serum BUN was markedly increased in HSPN mice.
The serum Cre was also increased in HSPN mice but without significance compared with the control mice. Compared with the HSPN model group, QZXB GR treatment might significantly improve the renal function in HSPN mice, by elevating the serum TP and ALB, decreasing the serum Cre and BUN. 
3.4. QZXB GR Suppressed the Immune Complexes IgA and IgG Deposition in HSPN Mice Kidneys. Immunohistochemistry analysis was used to investigate the effects of QZXB GR on the deposition of immune complex in renal tissues of HSPN mice. As shown in Figure 3, compared with the control mice, large amounts of IgA (Figures $3(\mathrm{~g})$ and $3(\mathrm{~h})$ ) and IgG (Figures 3(i) and 3(j)) deposition could be observed in the mesentery, vascular loop, tubular basement membrane (TBM), and arteriole wall in HSPN model mice $(P<0.01)$. While in contrast to which, QZXB GR treatment might significantly inhibit IgA and $\operatorname{IgG}$ deposition $(P<0.05$ or $<0.01$ ), as well as XN Caps and MMF treatment. Moreover, combination treatment showed a superimposed effect in renal IgA and IgG deposition in HSPN mice.

\subsection{QZXB GR Inhibited the Th2 Immunodeviation in HSPN} Mice. Flow cytometric analysis was used to analyze the lymphocyte subsets in peripheral blood and spleen in HSPN mice. As shown in Figure 4(a), the proportion of CD19+ B cells was markedly increased in peripheral blood of HSPN model mice $(P<0.05)$, while QZXB GR $(7.2 \mathrm{~g} / \mathrm{kg})$ treatment might significantly inhibit the increase $(P<0.05)$. However, no significance was observed in the proportion of $\mathrm{IL}^{+} \mathrm{T}$ cells and IFN- $\gamma^{+}$T cells in peripheral blood (data not shown).

Although there was no significance, the ratio of $\mathrm{IL}^{+}$ $\mathrm{T}$ cells/IFN- $\gamma^{+}$T cells was markedly elevated in HSPN mice, showing a Th2 immunodeviation in spleen lymphocyte subsets (Figure 4(b)), while QZXB GR treatment might significantly recovere the Th1/Th2 disorder, as well as $\mathrm{XN}$ Caps, MMF, and QZXB GR (7.2 g/kg) + MMF treatment.

\section{Discussion}

Nephritis is the principal cause of morbidity and mortality in HSP [17]. Although many therapies have been reported, their limited effectiveness and adverse effects highlight the urgent need for new drugs for HSPN treatment. However, to date, animal model of HSPN was rarely reported, which limits the advance research in the pathogenesis and treatment of HSPN. In this study, a HSPN mice model was established using Gliadin plus Indian Ink, referring to the IgAN model, for the similarity of immunological and pathological characteristics between HSPN and IgAN [13]. The dietary antigen Gliadin was applied to stimulate mucosal immune system, leading to the sustainable production of immunoglobulin in peripheral blood and the deposition of immunoglobulin in mesangial [18]. Indian Ink was used to block reticuloendothelial system (RES) and cause RES clearance dysfunction, which may prevent the clearance of IgA $[19,20]$. In this study, many features in the mice model were found to be consistent with characteristics of HSPN patients in clinical. Therefore, the mice model may partially be used as a tool for understanding the potential pathological mechanism and developing therapeutic strategy of HSPN.

Using the HSPN mice model, the effect and potential mechanism of QZXB GR were evaluated in this study. Moreover, XN caps (a TCM which consists of peanut seed coat extract for HSPN treatment in clinical $[18,21])$ and MMF (an immunosuppressive agent used to alleviate HSPN renal tissue injury [1]) were used as positive control drugs to ensure the sensitivity of this model. The results in this study suggested that QZXB GR was able to recover the renal injury from HSPN in mice, by alleviating renal pathological injury, inhibiting serum IL- 6 and CIC, as well as XN caps and MMF administration. Furthermore, in this study, the results also suggested that combinatory administration of QZXB GR and MMF might exhibit a superimposed effect in HSPN mice, which were consistent with that multiple drug combination therapy was effective in ameliorating proteinuria and pathological severity in clinical setting $[1,22]$.

In HSPN, the long-term prognosis is largely determined by the extent of renal pathological injury, characterized by mesangial injury, mesangial proliferation, crescent formation, and interstitial inflammation [22]. In this study, H\&E staining was used to determine the histopathologic pathological score changes in glomerular injury, renal tubal injury, and renal interstitial inflammation, whereas PAS staining used to observe the changes in glomerular basement membrane. The results showed that QZXB GR treatment significantly alleviated renal pathological injury and mesangial matrix proliferation in HSPN mice.

Petechiae and palpable purpura are the most common skin lesions in HSPN [1]. Purpura is often distributed over the surfaces of the lower legs, arms, and the sides of the buttocks, and subcutaneous bleeding may occur anywhere [23]. In this study, the subcutaneous hemorrhage on left legs in HSPN mice were observed and pictured. Our data showed that subcutaneous hemorrhage in HSPN mice was severe and might be significantly alleviated with QZXB GR treatment.

In this study, microscopic hematuria and renal biochemical parameters were used to determine the renal function changes in HSPN mice. The urinary RBC and WBC are always determined for assessing the HSPN patients' kidney function [24]. The increase in proteinuria is an important symbol of glomerular filtration barrier damage and reflects the severity of renal damage [25]. Serum ALB, $\mathrm{BUN}$, and Cre are classical biomarkers of renal function. Reduced serum ALB and increased serum BUN and Cre concentrations often indicate glomerular injuries, tubule injuries, and glomerular filtration rate decline [26]. The results of this study showed that QZXB GR treatment might significantly reduce the levels of proteinuria, urinary $\mathrm{RBC}$ and $\mathrm{WBC}$, increased serum TP and ALB, decreased serum BUN and Cre, implying to reverse the abnormality of these renal biochemical parameters in HSPN mice.

IL- 6 as a cytokine induces the maturation of B cells into immunoglobulin-secreting cells and promotes the survival and maintenance of long-lived plasma cells [27]. For serum IL-6 significantly increased in patients, it has been a valuable biomarker for clinical diagnosis of HSP [2]. Data in this study showed that the levels of serum IL-6 were significantly increased in HSPN mice, although QZXB GR treatment significantly prevented the increase, which also seemingly suggested that the beneficial effects of QZXB GR could be associated with its anti-inflammation and modulating B-cell maturation activity. 
Furthermore, to extend these findings, the underlying mechanisms on HSPN of QZXB GR were investigated. The clinical features of HSPN suggest that the disease is due to systemic deposition of CIC [28]. IgA is one of the most important risk factors in renal lesions development and progression in HSPN [29], which deposit in glomerular leading to cell proliferation, cytokine release, extracellular matrix production, and renal inflammatory changes [30], and in vessel walls, it may be responsible for symptoms involving the skin (petechiae and palpable purpura), joints, and kidneys $[30,31]$. The levels of IgG in children with HSPN are extremely high during purpura development, which deposits in mesangial might be associated with renal injury [13]. The results in this study showed that QZXB GR treatment could significantly decrease the levels of serum CIC and block IgA and IgG deposition in kidney of HSPN mice. These suggested that QZXB GR probably exert its renoprotective effects through reducing the production and deposition of immune complexes in the kidney and decreasing immune complex-mediated injury.

Also, it is generally believed that HSPN patients harbor a disorder in immune balance [32]. B cells are generally considered to take part in regulating the immune response, due to their ability to secrete antibodies (such as IgA, IgG, and IgE) and mediate humoral immune response [24]. In this study, QZXB GR decreased the proportion of CD19+ $B$ cells in peripheral blood and decreased the serum CIC, which was synthetized and secreted by B cells, implying that QZXB GR might reduce the number of B cells and inhibited its synthetic and secretory function. The balance between Th1 and Th2 cells in the progression of HSPN is still controversial. Some reported that a shift to Th1 cells existed in HSPN children [33], whereas others suggested that an excessive activation of Th2 lymphocytes and a decrease in Th1/ Th2 ratio existed in children with HSPN [14]. In addition, cytokines secreted by Th2 cells such as IL- 4 increased and the ratio of IL-4/IFN- $\gamma$ was elevated in HSPN patients [16], which may contribute to disease by activating B cells and enhancing immunoglobulin production $[34,35]$. The results of this study showed that QZXB GR treatment significantly reserved Th1/Th2 immune balance in HSPN mice, which suggested that QZXB GR probably exert its renoprotective effects partly through reversing Th2-dominated immune responses and maintaining immune balance by reducing the ratio of Th2/Th1.

In conclusion, based on the facts that QZXB GR reduced hematuria/proteinuria, reduced skin purpura, attenuated inflammation, and preserved renal function in HSPN mice by suppressing immune complexes deposition and Th2 immune deviation, we believe that it might be a potential drug candidate for HSPN treatment in clinical. Furthermore, the combination therapy of QZXB GR and MMF brought a light of hope for attenuating HSPN.

\section{Data Availability}

The data used to support the findings of this study are available from the corresponding author upon request.

\section{Disclosure}

Hui Yang, Jing Guan, and Mingbao Lin are co-first authors. The funders had no role in study design, data collection and analysis, decision to publish, or preparation of the manuscript.

\section{Conflicts of Interest}

There are no conflicts of interest concerning this work.

\section{Authors' Contributions}

HY, JG, and ML contributed equally to this work. HY and JG performed most experiments, analyzed the data, and wrote portions of the paper draft. PM, YNF, JYB, SYL, and JQY participated in some experiments. MBL, JMZ, and $\mathrm{QH}$ designed experiments, analyzed the data, and wrote the paper. MBL oversaw the overall execution of the projects and gave final approval of the version to be submitted.

\section{Acknowledgments}

This work was financially supported by the CAMS Initiative for Innovative Medicine (Grant no. 2016-I2M-2-006), the Beijing Natural Science Foundation Program (Grant no. 7182116), the Drug Innovation Major Project of China (Grant no. 2018ZX09711001-003-001), and National Natural Science Foundation of China (Grant no. 81703740).

\section{References}

[1] J.-Y. Chen and J.-H. Mao, "Henoch-Schönlein purpura nephritis in children: incidence, pathogenesis and management," World Journal of Pediatrics, vol. 11, no. 1, pp. 29-34, 2015.

[2] Z. Su, X. Lv, Y. Liu, J. Zhang, J. Guan, and Z. Gai, "Circulating midkine in children with Henoch-Schönlein purpura: clinical implications," International Immunopharmacology, vol. 39, pp. 246-250, 2016.

[3] A. Nickavar and M. Sadeghian, "Mycophenolate mofetil for the treatment of Henoch-Schonlein purpura nephritis; current knowledge and new concepts," Journal of Nephropathology, vol. 6, no. 3, pp. 103-104, 2017.

[4] J. Zhang, J. Lv, S. Pang et al., "Chinese herbal medicine for the treatment of Henoch-Schonlein purpura nephritis in children: a prospective cohort study protocol," Medicine (Baltimore), vol. 97, no. 24, Article ID e11064, 2018.

[5] W. Hong, D. Ying, and R. Qing, "Effect of tripterygium glycosides and administration of Chinese medicine based on differentiation on the coagulation mechanism of HenochSchonlein purpura nephritis in children," Journal of Traditional Chinese Medicine, vol. 53, pp. 212-214, 2012, in Chinese.

[6] S. Feng, "Integrative medicine treatment of children with allergic purpura nephritis," Chinese Archives of Traditional Chinese Medicine, vol. 31, pp. 1718-1720, 2013, in Chinese.

[7] D. Ding, H. Yan, and X. Zhen, "Effects of Chinese herbs in children with Henoch-Schonlein purpura nephritis: a randomized controlled trial," Journal of Traditional Chinese Medicine, vol. 34, no. 1, pp. 15-22, 2014. 
[8] Z. Tan, J. Guan, Y. Wang et al., "Optimization of alcohol extraction process of qingzixiaoban granules by orthogonal test," Beijing Medical Journal, vol. 39, no. 7, pp. 748-750, 2017, in Chinese.

[9] O. Nicoara and K. Twombley, "Immunoglobulin a nephropathy and immunoglobulin a vasculitis," Pediatric Clinics of North America, vol. 66, no. 1, pp. 101-110, 2019.

[10] C. Gomez-Guerrero, M. J. Lopez-Armada, E. Gonzalez, and J. Egido, "Soluble IgA and IgG aggregates are catabolized by cultured rat mesangial cells and induce production of TNFalpha and IL-6, and proliferation," Journal of Immunology, vol. 153 , no. 11 , pp. $5247-5255,1994$.

[11] D. J. Todd and S. M. Helfgott, "Serum sickness following treatment with rituximab," The Journal of Rheumatology, vol. 34, no. 2, pp. 430-433, 2007.

[12] J.-C. Davin and R. Coppo, "Henoch-Schönlein purpura nephritis in children," Nature Reviews Nephrology, vol. 10, no. 10, pp. 563-573, 2014.

[13] S. Mao, X. Xuan, Y. Sha et al., "Clinico-pathological association of Henoch-Schoenlein purpura nephritis and IgA nephropathy in children," International Journal of Clinical and Experimental Pathology, vol. 8, no. 3, pp. 2334-2342, 2015.

[14] Y.-y. Li, C.-r. Li, G.-b. Wang, and J. Yang, "Investigation of the change in CD4+ $\mathrm{T}$ cell subset in children with HenochSchonlein purpura," Rheumatology International, vol. 32, no. 12, pp. 3785-3792, 2012.

[15] N. Zu, U. Saatci, S. Ruacan et al., "The role of cytokines in Henoch Schonlein purpura," Scandinavian Journal of Rheumatology, vol. 26, no. 6, pp. 456-460, 1997.

[16] Y. X. Pan, Q. Ye, W. X. Shao, and S. Q. Shang, "Relationship between immune parameters and organ involvement in children with Henoch-Schonlein purpura," PLoS One, vol. 9, no. 12, Article ID e115261, 2014.

[17] P. Tarshish, J. Bernstein, and C. M. Edelmann, "HenochSchönlein purpura nephritis: course of disease and efficacy of cyclophosphamide," Pediatric Nephrology, vol. 19, no. 1, pp. 51-56, 2004.

[18] Z. Zhao, H. Fan, and H. Shi, "Pharmacodynamic study of Xuening on Henoch-Schonlein purpura nephritis mice model," Chinese Remedies \& Clinics, vol. 13, no. 8, pp. 1054-1055, 2013, in Chinese.

[19] R. Coppo, G. Mazzucco, G. Martina et al., "Gluten-induced experimental IgA glomerulopathy," Laboratory Investigation, vol. 60, no. 4, pp. 499-506, 1989.

[20] Z. Zheng, R. Ye, T. Jiang et al., "The effect of dietary antigen on IgA nephropathy," Academic Journal of Sun Yat-sen University of Medical Sciences, vol. 15, no. 1, pp. 29-32, 1994, in Chinese.

[21] H. Fan and Z. Zhao, "Clinical observation on treatment of henoch-schonlein purpura with xuening," Chinese Remedies \& Clinics, vol. 13, no. 11, pp. 1493-1495, 2013, in Chinese.

[22] H. Suzuki, R. Fan, Z. Zhang et al., "Aberrantly glycosylated $\operatorname{IgA} 1$ in IgA nephropathy patients is recognized by IgG antibodies with restricted heterogeneity," Journal of Clinical Investigation, vol. 119, no. 6, pp. 1668-1677, 2009.

[23] Y. Kawasaki, A. Ono, S. Ohara et al., "Henoch-Schönlein purpura nephritis in childhood: pathogenesis, prognostic factors and treatment," Fukushima Journal of Medical Science, vol. 59, no. 1, pp. 15-26, 2013.

[24] B. Yang, X. Tan, X. Xiong et al., "Effect of CD40/CD40L signaling on IL-10-producing regulatory B cells in Chinese children with Henoch-Schönlein purpura nephritis," Immunologic Research, vol. 65, no. 3, pp. 592-604, 2017.
[25] F. Y. Chow, D. J. Nikolic-Paterson, F. Y. Ma, E. Ozols, B. J. Rollins, and G. H. Tesch, "Monocyte chemoattractant protein-1-induced tissue inflammation is critical for the development of renal injury but not type 2 diabetes in obese $\mathrm{db} /$ db mice," Diabetologia, vol. 50, no. 2, pp. 471-480, 2007.

[26] W. Ge, H.-l. Wang, and R.-p. Sun, "Pentraxin 3 as a novel early biomarker for the prediction of Henoch-Schönlein purpura nephritis in children," European Journal of Pediatrics, vol. 173, no. 2, pp. 213-218, 2014.

[27] C. A. Hunter and S. A. Jones, "IL-6 as a keystone cytokine in health and disease," Nature Immunology, vol. 16, no. 5, pp. 448-457, 2015.

[28] M. Hilhorst, P. van Paassen, P. van Breda Vriesman, and J. W. C. Tervaert, "Immune complexes in acute adult-onset Henoch-Schonlein nephritis," Nephrology Dialysis Transplantation, vol. 26, no. 12, pp. 3960-3967, 2011.

[29] M. Pohl, "Henoch-Schönlein purpura nephritis," Pediatric Nephrology, vol. 30, no. 2, pp. 245-252, 2015.

[30] Q. Ye, S. Q. Shang, A. M. Liu et al., "24 h urinary protein levels and urine protein/creatinine ratios could probably forecast the pathological classification of HSPN," PLoS One, vol. 10, no. 5, Article ID e0127767, 2015.

[31] B. V. Reamy, P. M. Williams, and T. J. Lindsay, "HenochSchönlein purpura," American Family Physician, vol. 80, no. 7, pp. 697-704, 2009.

[32] N. Kondo, K. Kasahara, S. Shinoda, and T. Orii, "Accelerated expression of secreted? -chain gene in anaphylactoid purpura," Journal of Clinical Immunology, vol. 12, no. 3, pp. 193-196, 1992.

[33] K. Tsuruga, S. Watanabe, E. Oki et al., "Imbalance towards Th1 pathway predominance in purpura nephritis with proteinuria," Pediatric Nephrology, vol. 26, no. 12, pp. 2253-2258, 2011.

[34] I. Raphael, S. Nalawade, T. N. Eagar, and T. G. Forsthuber, "T cell subsets and their signature cytokines in autoimmune and inflammatory diseases," Cytokine, vol. 74, no. 1, pp. 5-17, 2015.

[35] J. A. Walker and A. N. J. McKenzie, "TH2 cell development and function," Nature Reviews Immunology, vol. 18, no. 2, pp. 121-133, 2018. 


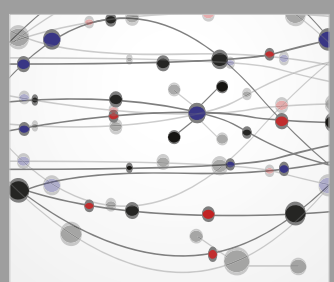

The Scientific World Journal
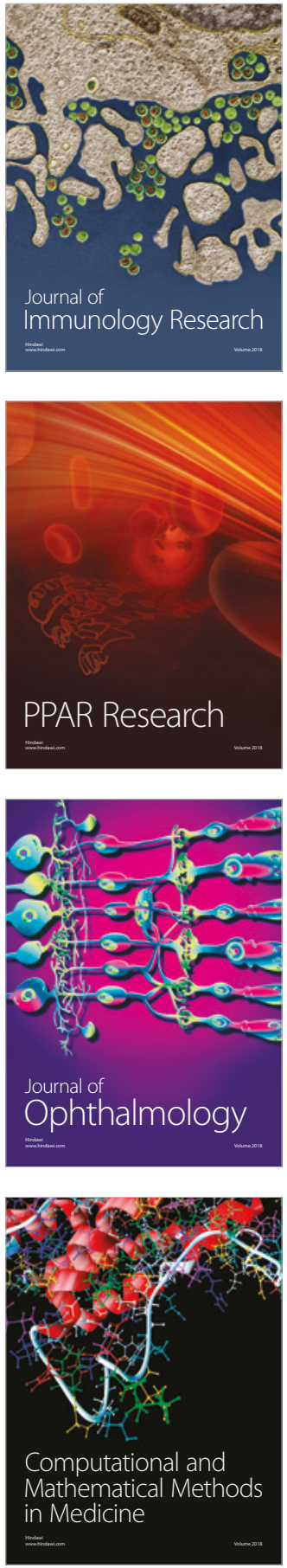

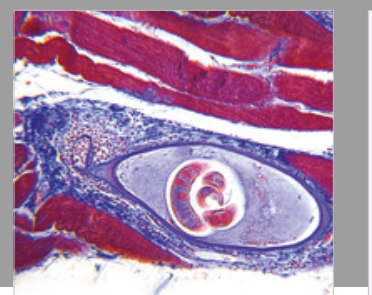

Gastroenterology Research and Practice

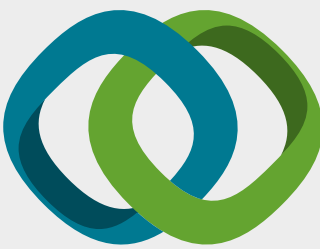

\section{Hindawi}

Submit your manuscripts at

www.hindawi.com
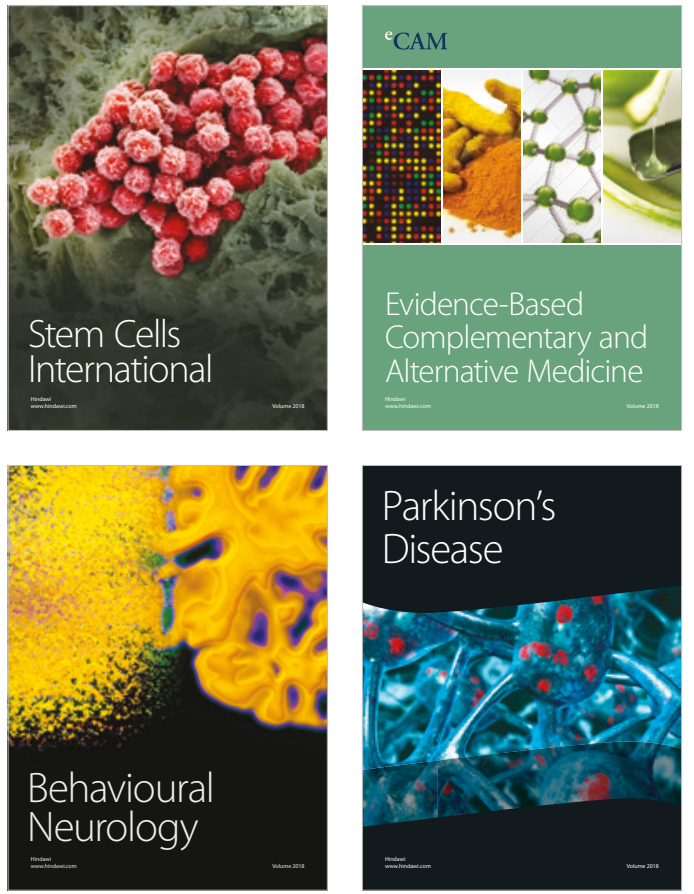

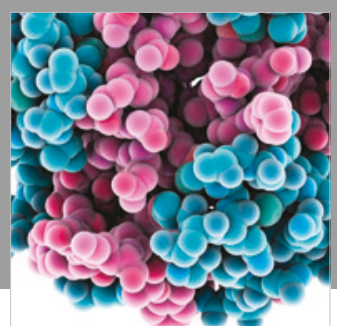

ournal of

Diabetes Research

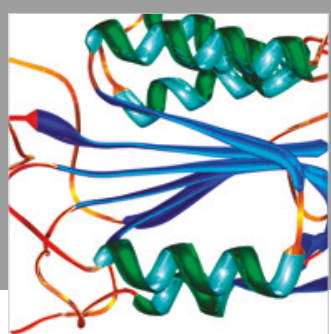

Disease Markers
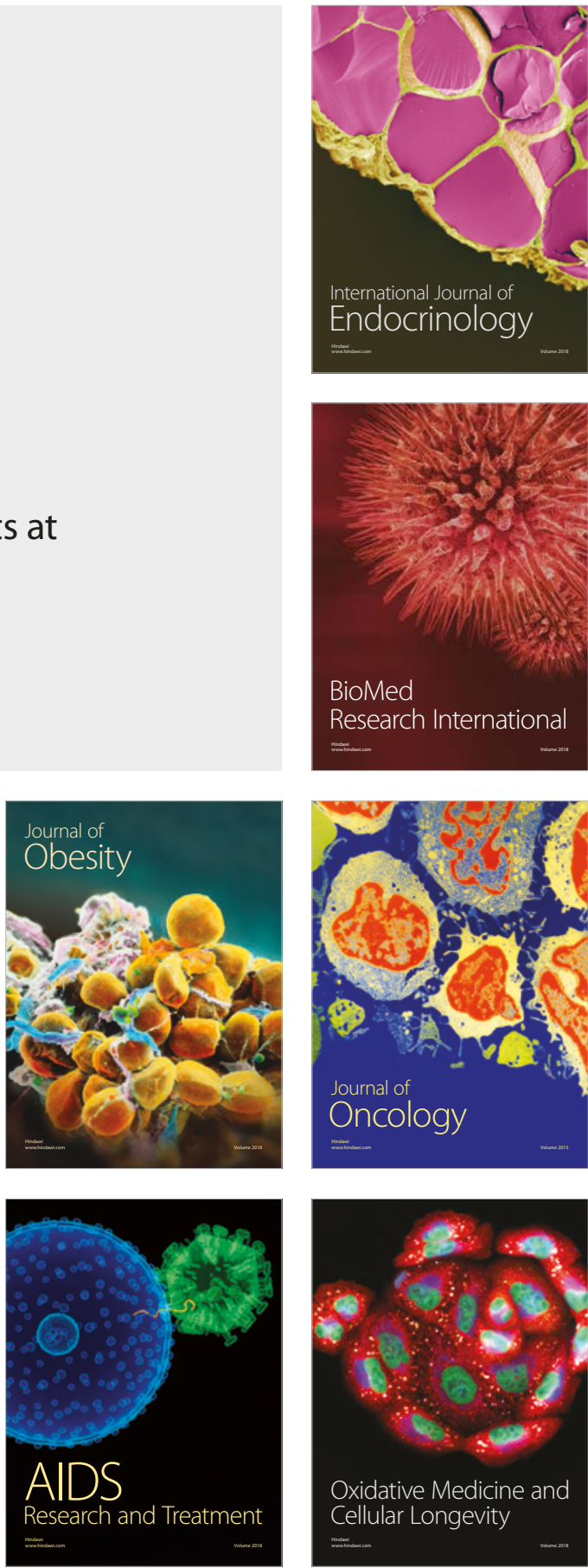